\title{
An Improved Description of Technetium Spectra (Tc I and Tc II), 2000 to $9000 \AA$
}

\author{
W. R. Bozman, W. F. Meggers, and C. H. Corliss \\ Institute for Basic Standards, National Bureau of Standards, Washington, D.C. 20234
}

(June 26, 1967)

\begin{abstract}
A new description of technetium spectra has been made that is more complete and provides more accurate data on wavelengths than those reported heretofore. The observations were made in the region 2000 to $9000 \AA$ with arc and spark sources. Larger samples of technetium and spectrographs of higher resolution were used and the number of lines reported (4500) is more than doubled. The intensities are estimated over a range from 1 to 20,000 and the relative strengths in are and spark permit assignment of the origin of the lines to neutral atoms (3300 lines) or ions (1200 lines).
\end{abstract}

Key Words: Spectra of technetium; technetium, spectra of; wavelengths of technetium

\section{Introduction}

It is nearly a hundred years since Mendeléeff predicted the existence of the chemical analog of manganese, which we now call technetium. Because it was first produced artificially, the element was named from the Greek work $\tau \epsilon \chi \nu \iota \kappa o ̀$, artificial [1]. ${ }^{1}$ Over the past 30 years increasingly larger amounts of the element have been artificially prepared. By 1949 Meggers and Scribner were able to obtain $6 \mathrm{mg}$ of ${ }^{99} \mathrm{Tc}$ from the Atomic Energy Commission. With this sample they made a description of arc and spark spectra of technetium [2] which they hoped would "serve for spectrochemical identification and for structural analyses of these spectra."

Although the list of lines published by Meggers and Scribner did not entirely fulfill their hopes, it provided material for a preliminary analysis of the structures of TCI and TCII by Meggers [3] and for the remarkable discovery by Merrill [4] of naturally occurring technetium in $\mathrm{R}$ Andromedae and other S stars.

In order to make a substantial extension to the preliminary analyses of Meggers [3] it has been necessary to reobserve the spectrum with larger samples of technetium and higher spectrographic resolution. The new observations, which are reported in the present paper, have doubled the number of known lines and significantly improved the accuracy of the wavelengths.

Using this new list of lines, and also observations of the Zeeman effect, Bozman [5, 6] has reported the classification of 900 lines of Tc I as transitions among

\footnotetext{
${ }^{1}$ Figures in brackets indicate the literature references at the end of this paper.
}

40 even and 70 odd levels. Most of these levels have been compiled and published by C. E. Moore [7]. Further analysis and details of the work already reported will be published shortly.

Moore and Catalán extended the analysis of Tc II from this list and provided new terms and $g$-values for her compilation, Atomic Energy Levels [8]. The analysis of TC II is far from complete. Further observations below $2000 \AA$ are needed.

\section{Observations}

\subsection{Samples and Sources}

These new observations were made possible by the generous loan of $20 \mathrm{mg}$ of ${ }^{99} \mathrm{Tc}$ as ammonium pertechnetate $\left(\mathrm{NH}_{4} \mathrm{TcO}_{4}\right)$ from the Atomic Energy Commission, obtained with the help of G. W. Parker of the Oak Ridge National Laboratory.

Preliminary experiments in the manipulation and excitation of the sample were conducted with $\left(\mathrm{NH}_{4}\right)_{6} \mathrm{Mo}_{7} \mathrm{O}_{24}$ because of the rarity and radioactivity of the technetium. As a result of studies of various electrical parameters and electrode configurations the following conditions were adopted for the exposures.

For the arc exposures $400 \mu \mathrm{g}$ of Tc was evaporated from solution in a shallow cup on the end of a $1 / 4$-in diam copper or silver rod which served as the lower anode. The upper cathode of the same material was tipped with a $90^{\circ}$ cone. A 2 -min exposure was made from the center portion of a $5 \mathrm{~A}$ arc in air drawn across a $3-\mathrm{mm}$ gap between the electrodes. 
For the spark exposures $200 \mu \mathrm{g}$ of Tc was evaporated from solution on the flat ends of a pair of truncated wedges forming the tips of $1 / 4$-in diam copper or silver rods. A 30-sec exposure was made with light from the portion of a 5-mm gap next to one electrode surface. The spark was produced by the discharge from a capacitor of $0.006 \mu \mathrm{F}$ charged by a $30,000 \mathrm{~V}$ transformer. Several successive exposures from freshly loaded pairs of electrodes were necessary to obtain spark spectra of adequate strength on the plates.

All regions of the spectrum were photographed with both silver and copper electrodes to permit observation of $\mathrm{Tc}$ lines masked by lines of the electrode material. A typical spectrogram consisted of successive juxtaposed spectra as follows: standard iron arc, $\mathrm{Cu}$ or Ag blank arc, arc of $\mathrm{Tc}$ on $\mathrm{Cu}$ or $\mathrm{Ag}$, spark of $\mathrm{Tc}$ on $\mathrm{Cu}$ or $\mathrm{Ag}, \mathrm{Cu}$ or $\mathrm{Ag}$ blank spark, standard iron arc.

The ares and sparks were always operated in a brass housing with quartz windows to prevent contamination of the surroundings with the radioactive ${ }^{99} \mathrm{Tc}$. A current of air was drawn through the housing with a vacuum pump and the exhaust was filtered through glass wool where the used Tc was collected.

\subsection{Spectrographs and Standards}

The region from 2000 to $6900 \AA$ was photographed with a 1200 line $/ \mathrm{mm}$ concave grating ruled by $\mathrm{R}$. W. Wood and mounted in parallel light. This mounting produces stigmatic images of the slit to allow use of juxtaposed spectra. The average resolution with the slit width used was about $1 / 50,000$, i.e., the equivalent slit width was about $0.06 \AA$ at $3000 \AA$. This region was photographed on Eastman 103-O-UV and 103a-F(3) plates. One set of exposures was made on the Hilger E-1 Littrow quartz prism spectrograph in the region 2000 to $2300 \mathrm{~A}$.

The region from 6600 to $9000 \AA$ was observed with a 600 line/mm grating mounted in parallel light and with an equivalent slit width of about 0.2 or $0.3 \AA$. No spark exposures were made in this region.

A few exposures were made beyond $9000 \AA$ and below $2000 \AA$ by using electrodeless lamps but the lamps did not run well.

Every region of the spectrum was photographed from two to four times and each plate was measured twice, each time by a different observer. Each wavelength represents about five measurements, on the average.

Since this work was all done in 1952 and 1953 before the advent of standard wavelengths from thorium, the iron arc was used to provide the standard lines.

\section{Results}

The results of this investigation are presented in table 1 , where wavelengths and relative intensities of 4500 spectral lines characteristic of technetium atoms and ions are given. In the region from 2000 to $2300 \AA$ the wavelengths represent the average of eight measurements for most of the lines. From 2300 to
$4200 \AA$, six observations were made and from 4200 to $9000 \AA$ the averages were made from four observations. All regions were observed in both arc and spark and on both copper and silver electrodes except for wavelengths longer than $6900 \AA$ where no spark observations were made.

In the earlier experiments reported in [2], all the spectra were excited from copper electrodes; in the present work the use of silver electrodes as well as higher resolving power has revealed lines previously obscured by copper lines. In particular, the $T_{c}$ II line at 2529.34 was not previously resolved from the $\mathrm{Cu}$ II line at 2529.30 and its absence from the earlier line list prevented the discovery of the $4 d^{6} a^{5} \mathrm{D}$ term of Te II by Meggers [3].

The probable error in the wavelengths depends, of course, on the region of the spectrum and on the number of measurements. The agreement observed among the measurements was sufficient to warrant the retention of three decimal places at wavelengths shorter than $6900 \AA$; at longer wavelengths only two decimal places are given.

The relative intensities of the lines have been estimated on a scale extending from 1 to 20,000 . The relative intensities of the very strong lines are undoubtedly distorted by severe self-absorption and the true relative intensities among these lines are better represented by the numbers reported in reference [2], where a smaller amount of Tc was used in each exposure. No attempt has been made to represent a true scale of relative power radiated in different regions of the spectrum. It is probable that the intensities in the short wavelength regions, expecially those below $2300 \AA$ are much underestimated relative to the visible and near ultraviolet, whereas intensities in the infrared portion are probably slightly overestimated.

The lines which are stronger in the arc originate in the neutral atom and those of equal or greater intensity in the spark originate in the singly charged ion or, perhaps in a few cases in the shortest wavelength region, in the doubly charged ion.

The intensity numbers are in some cases accompanied by literal symbols indicating characteristic features of the lines as follows:

$$
\begin{aligned}
& c \text {-complex } \\
& d \text {-double } \\
& e \text {-enhanced at the electrode } \\
& h \text {-hazy } \\
& H \text { - very hazy } \\
& l \text {-shaded to longer waves } \\
& s \text { - shaded to shorter waves } \\
& w \text { - wide } \\
& W \text { - very wide } \\
& t r \text {-intensity }<1 .
\end{aligned}
$$

The complex characteristic exhibited by some $\mathrm{Tc}_{\mathrm{c}}$ lines is due to hyperfine structure arising from interaction of valence electrons with the atomic nucleus. This structure has been studied in detail at higher resolution by Kessler and Trees [9]. 
TABLE I. Spectrum of technetium

\begin{tabular}{|c|c|c|c|c|c|c|c|c|c|c|c|}
\hline \multirow{2}{*}{$\begin{array}{l}\text { Wave- } \\
\text { length }\end{array}$} & \multicolumn{2}{|c|}{ Intensity } & \multirow{2}{*}{$\begin{array}{l}\text { Wave- } \\
\text { length }\end{array}$} & \multicolumn{2}{|c|}{ Intensity } & \multirow{2}{*}{$\begin{array}{l}\text { Wave- } \\
\text { length }\end{array}$} & \multicolumn{2}{|c|}{ Intensity } & \multirow{2}{*}{$\begin{array}{l}\text { Wave- } \\
\text { length }\end{array}$} & \multicolumn{2}{|c|}{ Intensity } \\
\hline & Arc & Spark & & Arc & Spark & & Arc & Spark & & Arc & Spark \\
\hline $\begin{array}{l}2054.468 \\
2057.704 \\
2058.880 \\
2060.266 \\
2060.961 \\
2062.929 \\
2064.022 \\
2064.211 \\
2064.853 \\
2065.154\end{array}$ & $\begin{array}{l}2 \\
2 \\
2 \mathrm{e} \\
4 \\
\\
\quad \mathrm{tr}\end{array}$ & $\begin{array}{l}4 \\
\\
2 \\
6 \\
2 \mathrm{e} \\
1 \\
1 \\
1\end{array}$ & $\begin{array}{l}2140.738 \\
2140.943 \\
2141.830 \\
2144.900 \\
2145.322 \\
2147.193 \\
2148.305 \\
2149.764 \\
2151.250 \\
2153.390\end{array}$ & $\begin{array}{l}1 \\
2 \\
5 \\
2 \\
4\end{array}$ & $\begin{array}{l}2 \\
4 \\
2 \\
5\end{array}$ & $\begin{array}{l}2258.410 \\
2260.870 \\
2261.247 \\
2263.533 \\
2266.220 \\
2267.424 \\
2268.100 \\
2270.722 \\
2271.094 \\
2271.218\end{array}$ & $\begin{array}{r}3 \\
10 \\
2\end{array}$ & $\begin{array}{r}1 \\
1 \\
15 \\
2 \\
30 \\
2 \\
1 \\
1 \\
50 \\
40\end{array}$ & $\begin{array}{l}2376.040 \\
2377.770 \\
2382.098 \\
2382.386 \\
2382.992 \\
2384.650 \\
2385.338 \\
2386.030 \\
2387.188 \\
2392.012\end{array}$ & $\begin{array}{l}1 \\
6 \\
3 \\
1\end{array}$ & $\begin{array}{r}2 \\
15 \\
2 \\
1\end{array}$ \\
\hline $\begin{array}{l}2067.630 \\
2068.323 \\
2071.289 \\
2072.072 \\
2073.563 \\
2076.700 \\
2077.210 \\
2079.071 \\
2079.504 \\
2079.808\end{array}$ & $\begin{array}{l}1 \\
1 \\
2\end{array}$ & $\begin{array}{r}3 \\
\\
4 \\
1 \\
1 \\
6 \\
10 \\
1\end{array}$ & $\begin{array}{l}2154.668 \\
2155.278 \\
2156.272 \\
2156.608 \\
2156.908 \\
2157.780 \\
2157.800 \\
2158.702 \\
2159.292 \\
2159.843\end{array}$ & $\begin{array}{c}2 \\
30 \\
1 \\
1 \\
2 \\
5 \mathrm{e}\end{array}$ & $\begin{array}{c}12 \\
1 \\
1 \\
2 \\
10 \\
\\
3 \\
50 \mathrm{~h}\end{array}$ & $\begin{array}{l}2271.662 \\
2272.441 \\
2272.976 \\
2274.925 \\
2274.624 \\
2278.403 \\
2280.304 \\
2280.884 \\
2282.121 \\
2282.361\end{array}$ & $\begin{array}{r}3 \\
5 \\
2 \\
2 \\
2 \\
1 \\
2 \\
4 \\
10\end{array}$ & 10 & $\begin{array}{l}2393.422 \\
2394.498 \\
2394.777 \\
2394.974 \\
2395.291 \\
2396.628 \\
2396.984 \\
2397.400 \\
2398.652 \\
2398.682\end{array}$ & 6 & $\begin{array}{l}1 \\
2 \\
1 \\
1 \\
2 \\
1 \\
2 \\
\\
60\end{array}$ \\
\hline $\begin{array}{l}2081.466 \\
2084.088 \\
2084.683 \\
2086.853 \\
2087.628 \\
2089.268 \\
2089.657 \\
2090.682 \\
2090.987 \\
2091.730\end{array}$ & $\begin{array}{l}5 \\
1 \\
1 \\
3 \\
3 \\
\\
4\end{array}$ & $\begin{array}{r}10 \\
3 \\
20 \\
2 \\
1\end{array}$ & $\begin{array}{l}2160.606 \\
2160.771 \\
2163.546 \\
2164.988 \\
2165.902 \\
2168.554 \\
2170.123 \\
2170.546 \\
2173.446 \\
2177.980\end{array}$ & $\begin{array}{l}2 \\
3 \\
5 \\
\\
1 \\
1 \\
3\end{array}$ & $\begin{array}{r}1 \\
10 \\
4 \\
8 \\
10 \\
1 \\
10 \\
\\
5 \\
4\end{array}$ & $\begin{array}{l}2282.713 \\
2285.451 \\
2287.470 \\
2287.931 \\
2291.637 \\
2292.104 \\
2298.080 \\
2299.095 \\
2299.947 \\
2300.405\end{array}$ & $\begin{array}{r}10 \\
50 \\
2 \\
1 \\
1 \\
1 \\
100 \\
1\end{array}$ & $\begin{array}{c}15 \\
1 \\
1 \\
3 \\
2 \\
30 \\
1 \\
2 \\
2 \mathrm{~h}\end{array}$ & $\begin{array}{l}2399.368 \\
2400.896 \\
2404.101 \\
2405.128 \\
2406.651 \\
2407.099 \\
2407.875 \\
2408.158 \\
2408.815 \\
2410.193\end{array}$ & $\begin{array}{l}4 \\
2 \\
8\end{array}$ & $\begin{array}{r}4 \\
50 \\
\\
3 \\
3 \\
6 \\
6 \\
2\end{array}$ \\
\hline $\begin{array}{l}2092.830 \\
2093.310 \\
2094.551 \\
2096.307 \\
2096.519 \\
2098.119 \\
2098.756 \\
2099.330 \\
2099.680 \\
2101.520\end{array}$ & $\begin{array}{l}1 \\
1 \\
2 \\
2 \\
3 \\
5 \\
2 \\
1 \\
\\
4\end{array}$ & $\begin{array}{r}1 \\
10\end{array}$ & $\begin{array}{l}2178.935 \\
2180.454 \\
2182.032 \\
2183.001 \\
2184.244 \\
2185.394 \\
2187.476 \\
2188.218 \\
2189.055 \\
2191.623\end{array}$ & $\begin{array}{r}30 \\
1 \\
5 \\
30 \\
2\end{array}$ & $\begin{array}{r}8 \\
6 \\
30 \\
15 \\
2 \\
\\
1 \\
20 \\
\\
\end{array}$ & $\begin{array}{l}2300.950 \\
2302.556 \\
2310.706 \\
2313.897 \\
2318.731 \\
2324.596 \\
2328.389 \\
2334.046 \\
2336.041 \\
2337.526\end{array}$ & 3 & $\begin{array}{l}4 \\
1 \\
2 \mathrm{~h} \\
1 \\
3 \\
\\
2 \\
1 \\
2 \\
2\end{array}$ & $\begin{array}{l}2412.610 \\
2414.471 \\
2416.217 \\
2417.524 \\
2418.031 \\
2418.647 \\
2418.737 \\
2419.818 \\
2420.775 \\
2421.296\end{array}$ & $\begin{array}{r}6 \\
30 \\
7 \\
5 \\
2 \\
2 \mathrm{~h}\end{array}$ & $\begin{array}{l}3 \\
3 \\
1 \\
1 \\
2 \mathrm{~h}\end{array}$ \\
\hline $\begin{array}{l}2101.580 \\
2101.811 \\
2103.808 \\
2104.054 \\
2104.340 \\
2105.248 \\
2106.234 \\
2107.617 \\
2107.848 \\
2108.680\end{array}$ & $\begin{array}{l}1 \\
6\end{array}$ & $\begin{array}{r}1 \\
\\
1 \\
1 \\
2 \\
30 \\
1 \\
6 \\
1\end{array}$ & $\begin{array}{l}2193.347 \\
2193.999 \\
2196.669 \\
2197.501 \\
2198.344 \\
2198.517 \\
2199.825 \\
2204.151 \\
2206.006 \\
2207.310\end{array}$ & $\begin{array}{l}4 \\
2\end{array}$ & $\begin{array}{r}1 \\
20 \\
4 \\
15 \\
2 \\
1 \\
8\end{array}$ & $\begin{array}{l}2338.278 \\
2341.028 \\
2341.610 \\
2341.970 \\
2344.719 \\
2344.845 \\
2345.088 \\
2345.162 \\
2346.592 \\
2349.167\end{array}$ & $\begin{array}{l}1 \\
2\end{array}$ & $\begin{array}{l}3 w \\
2 \\
6 \\
1 \\
10 \\
6 \\
7 \\
5 \\
5\end{array}$ & $\begin{array}{l}2423.228 \\
2424.538 \\
2425.250 \\
2425.904 \\
2427.084 \\
2427.848 \\
2431.360 \\
2432.337 \\
2432.596 \\
2433.728\end{array}$ & $\begin{array}{l}50 \\
20 \\
\\
\\
2 \\
3 \\
\\
2 \\
\\
8\end{array}$ & $\begin{array}{l}3 \\
1 \\
1 \\
1 \\
1 \\
2 \mathrm{~h}\end{array}$ \\
\hline $\begin{array}{l}2109.056 \\
2110.302 \\
2111.252 \\
2114.259 \\
2114.484 \\
2116.436 \\
2118.218 \\
2119.114 \\
2119.406 \\
2123.830\end{array}$ & $\begin{array}{r}1 \\
5 \\
5 \\
3 \\
5 \\
20 \\
2 \\
4 \\
15 \\
3\end{array}$ & $\begin{array}{c}4 \mathrm{~h} \\
\\
30 \\
2\end{array}$ & $\begin{array}{l}2214.224 \\
2215.245 \\
2216.031 \\
2216.244 \\
2221.000 \\
2221.133 \\
2221.349 \\
2221.918 \\
2226.626 \\
2227.876\end{array}$ & 3 & $\begin{array}{l}2 \mathrm{~h} \\
20 \\
4 \\
8 \\
2 \\
3 \\
4 \\
6 \\
6\end{array}$ & $\begin{array}{l}2349.594 \\
2350.096 \\
2350.320 \\
2353.166 \\
2353.742 \\
2354.096 \\
2354.448 \\
2355.979 \\
2356.839 \\
2360.456\end{array}$ & 4 & $\begin{array}{l}1 \\
1 \\
1 \\
2 \\
3 \\
3 \\
1 \\
1 \\
1\end{array}$ & $\begin{array}{l}2434.364 \\
2434.859 \\
2435.628 \\
2435.829 \\
2436.824 \\
2436.994 \\
2439.081 \\
2441.284 \\
2441.494 \\
2445.560\end{array}$ & $\begin{array}{r}20 \\
10 \\
5 \\
\\
1 \\
1\end{array}$ & $\begin{array}{l}2 \\
1 \\
1 \\
5\end{array}$ \\
\hline $\begin{array}{l}2124.074 \\
2125.302 \\
2126.542 \\
2130.284 \\
2131.912 \\
2132.061 \\
2133.182 \\
2133.658 \\
2135.169 \\
2138.133\end{array}$ & $\begin{array}{l}1 \\
4 \mathrm{~h} \\
3\end{array}$ & $\begin{array}{c}30 \mathrm{~h} \\
1 \mathrm{~h} \\
2 \\
5 \\
4 \\
2 \\
30 \mathrm{~h} \\
6 \mathrm{~h}\end{array}$ & $\begin{array}{l}2233.860 \\
2234.642 \\
2236.270 \\
2237.176 \\
2237.466 \\
2237.919 \\
2248.533 \\
2252.319 \\
2254.524 \\
2256.790\end{array}$ & $\begin{array}{l}6 \\
1\end{array}$ & $\begin{array}{l}8 \\
10 \mathrm{w} \\
6 \\
3 \\
1 \\
3 \\
\\
1 \\
3 \mathrm{hw} \\
5\end{array}$ & $\begin{array}{l}2360.770 \\
2361.827 \\
2365.533 \\
2365.856 \\
2366.539 \\
2367.660 \\
2368.010 \\
2368.349 \\
2369.026 \\
2372.104\end{array}$ & 5 & $\begin{array}{l}8 \\
1 \\
1 \\
2 \\
6 \\
1 \\
1 \\
2 \\
2 \\
1\end{array}$ & $\begin{array}{l}2446.049 \\
2446.864 \\
2447.440 \\
2447.860 \\
2447.900 \\
2448.603 \\
2448.950 \\
2449.119 \\
2450.093 \\
2450.614\end{array}$ & $\begin{array}{l}3 \\
4 \\
3\end{array}$ & $\begin{array}{c}1 \\
2 \\
2 \\
10 w \\
4 \\
6\end{array}$ \\
\hline
\end{tabular}


TABLE I. Spectrum of technetium - Continued

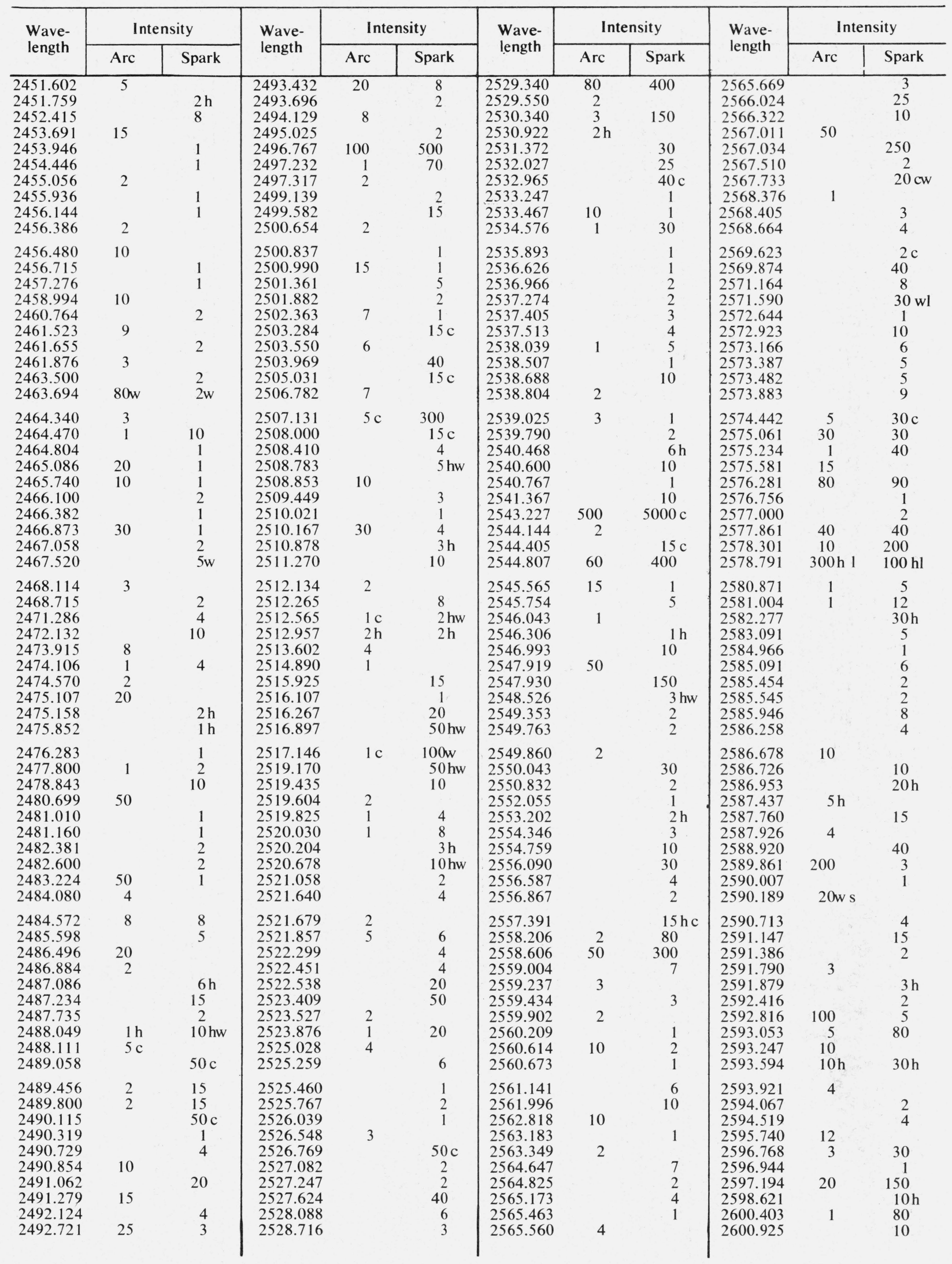


TABLE I. Spectrum of technetium - Continued

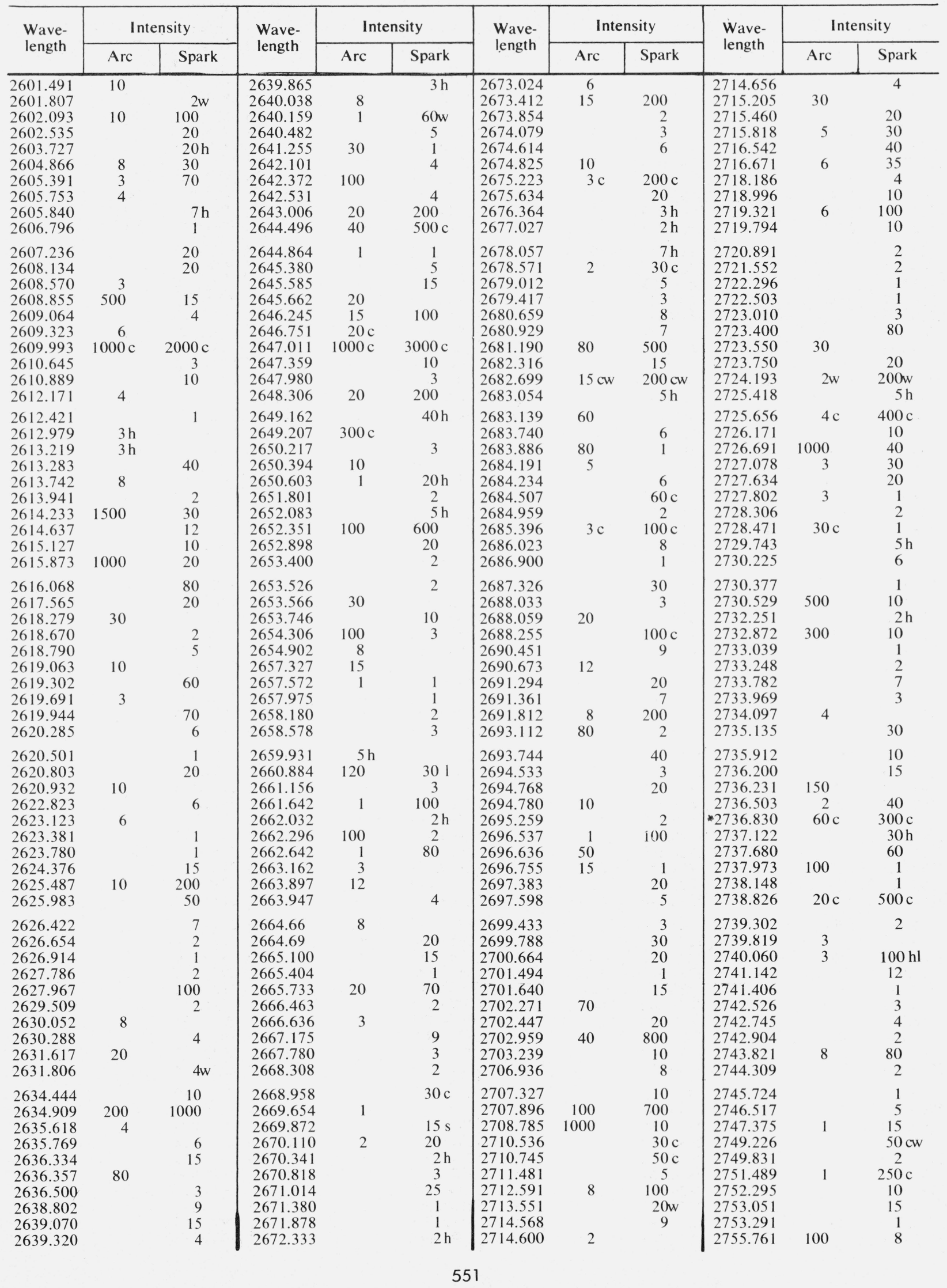


TABLE I. Spectrum of technetium - Continued

\begin{tabular}{|c|c|c|c|c|c|c|c|c|c|c|c|}
\hline \multirow{2}{*}{$\begin{array}{l}\text { Wave- } \\
\text { length }\end{array}$} & \multicolumn{2}{|c|}{ Intensity } & \multirow{2}{*}{$\begin{array}{l}\text { Wave- } \\
\text { length }\end{array}$} & \multicolumn{2}{|c|}{ Intensity } & \multirow{2}{*}{$\begin{array}{l}\text { Wave- } \\
\text { length }\end{array}$} & \multicolumn{2}{|c|}{ Intensity } & \multirow{2}{*}{$\begin{array}{l}\text { Wave- } \\
\text { length }\end{array}$} & \multicolumn{2}{|c|}{ Intensity } \\
\hline & Arc & Spark & & Arc & Spark & & Arc & Spark & & Arc & Spark \\
\hline $\begin{array}{l}2757.008 \\
2757.282 \\
2757.577 \\
2758.714 \\
2759.211 \\
2759.790 \\
2760.132 \\
2760.380 \\
2761.281 \\
2762.134\end{array}$ & $\begin{array}{r}7 \\
100\end{array}$ & $\begin{array}{c}1 \\
6 \\
6 \\
5 \\
1 \\
2 \\
15 \mathrm{~h} \\
\\
8 \\
3\end{array}$ & $\begin{array}{l}2795.286 \\
2795.650 \\
2795.778 \\
2796.709 \\
2797.730 \\
2798.079 \\
2798.209 \\
2800.450 \\
2800.770 \\
2802.455\end{array}$ & $\begin{array}{r}80 \\
200 \\
4 \\
3 \\
2 \\
8\end{array}$ & $\begin{array}{r}2 \\
500 \\
80 \\
10 \\
300 \\
\\
4 \\
10 \\
2\end{array}$ & $\begin{array}{l}2838.547 \\
2838.876 \\
2839.140 \\
2839.854 \\
2840.375 \\
2841.656 \\
2842.303 \\
2843.352 \\
2843.766 \\
2845.044\end{array}$ & $\begin{array}{r}8 \\
60\end{array}$ & $\begin{array}{c}50 \mathrm{c} \\
8 \\
1 \\
30 \\
400 \\
1 \\
4 \\
20 \\
3\end{array}$ & $\begin{array}{l}2883.508 \\
2883.670 \\
2884.748 \\
2885.548 \\
2886.079 \\
2887.429 \\
2887.734 \\
2888.458 \\
2889.200 \\
2889.487\end{array}$ & $\begin{array}{r}1 \\
1000 \\
100 \\
30 \\
3\end{array}$ & $\begin{array}{c}5 \\
10 \\
20 \mathrm{c} \\
20 \\
2 \\
8 \\
25 \\
300 \mathrm{c}\end{array}$ \\
\hline $\begin{array}{l}2762.337 \\
2763.231 \\
2763.871 \\
2763.957 \\
2764.501 \\
2765.507 \\
2765.713 \\
2765.947 \\
2766.886 \\
2767.363\end{array}$ & $\begin{array}{r}200 \\
8\end{array}$ & $\begin{array}{c}8 \\
50 \\
5 \\
\\
5 \\
3 \\
20 \mathrm{c} \\
1 \\
10 \\
10 \mathrm{~h}\end{array}$ & $\begin{array}{l}2802.810 \\
2803.018 \\
2803.389 \\
2803.921 \\
2804.855 \\
2805.179 \\
2805.983 \\
2806.626 \\
2807.100 \\
2807.433\end{array}$ & $\begin{array}{r}1000 \\
150 \\
8 \\
6\end{array}$ & $\begin{array}{r}8 \\
3 \\
4 \\
\\
\\
10 \\
2 \\
15 \\
2 \\
3\end{array}$ & $\begin{array}{l}2845.472 \\
2845.786 \\
2846.392 \\
2846.617 \\
2846.742 \\
2847.327 \\
2847.700 \\
2847.874 \\
2847.909 \\
2848.157\end{array}$ & $\begin{array}{r}7 \\
2 \\
10 \\
10 \\
6 \\
\\
1\end{array}$ & $\begin{array}{r}4 \\
3 \\
200 \\
3 \\
30 \\
\\
5 w \\
\\
2 \\
4 w\end{array}$ & $\begin{array}{l}2890.438 \\
2890.793 \\
2891.176 \\
2891.324 \\
2892.347 \\
2892.759 \\
2893.160 \\
2893.448 \\
2893.900 \\
2894.322\end{array}$ & $\begin{array}{r}4 \\
200 \\
150 \\
\\
200\end{array}$ & $\begin{array}{c}20 \\
300 \mathrm{c} \\
5 \\
15 \\
60 \\
150 \\
2 \\
2 \\
10 \\
2\end{array}$ \\
\hline $\begin{array}{l}2769.051 \\
2769.590 \\
2769.714 \\
2769.879 \\
2771.216 \\
2771.797 \\
2772.052 \\
2773.303 \\
2773.786 \\
2774.278\end{array}$ & $\begin{array}{c}15 \\
5 \\
2 \mathrm{~h} \\
1 \\
2\end{array}$ & $\begin{array}{c}4 \\
15 \\
5 \\
5 \\
100 \mathrm{c} \\
10 \\
6 \\
30 \\
200 \\
4\end{array}$ & $\begin{array}{l}2807.915 \\
2808.356 \\
2809.646 \\
2810.046 \\
2810.225 \\
2810.240 \\
2811.152 \\
2811.614 \\
2812.447 \\
2812.999\end{array}$ & $\begin{array}{c}500 \\
50 \mathrm{c} \\
8 \\
15 \\
\\
500 \\
3\end{array}$ & $\begin{array}{c}15 \mathrm{~d} \\
10 \\
150 \mathrm{c} \\
\\
20 \\
1 \mathrm{~h} \\
1000 \\
4\end{array}$ & $\begin{array}{l}2849.196 \\
2850.154 \\
2850.931 \\
2850.956 \\
2851.222 \\
2851.358 \\
2851.732 \\
2851.985 \\
2852.294 \\
2853.319\end{array}$ & $\begin{array}{r}60 \\
150 \\
1\end{array}$ & $\begin{array}{c}3 \\
2 \\
10 \\
10 \\
3 \\
3 \\
4 \\
20 \mathrm{w} \\
15 \\
2\end{array}$ & $\begin{array}{l}2894.831 \\
2895.473 \\
2896.340 \\
2897.776 \\
2899.363 \\
2900.692 \\
2902.134 \\
2902.539 \\
2902.910 \\
2903.813\end{array}$ & $\begin{array}{r}1000 \\
6\end{array}$ & $\begin{array}{r}15 \\
15 \\
10 \\
2 \\
2 \\
80 \\
10 \\
4 \\
4 \\
3\end{array}$ \\
\hline $\begin{array}{l}2774.809 \\
2775.076 \\
2775.328 \\
2775.601 \\
2776.041 \\
2776.559 \\
2777.313 \\
2777.645 \\
2778.117 \\
2778.269\end{array}$ & $\begin{array}{l}6 \\
3\end{array}$ & $\begin{array}{c}1 \mathrm{~h} \\
10 \\
70 \\
10 \\
4 \\
500 \\
2 \\
9 \\
1\end{array}$ & $\begin{array}{l}2813.499 \\
2813.784 \\
2814.184 \\
2814.626 \\
2814.847 \\
2814.860 \\
2814.959 \\
2815.965 \\
2816.515 \\
2816.999\end{array}$ & $\begin{array}{r}8 \\
30 \\
2 \\
3\end{array}$ & $\begin{array}{c}5 \mathrm{~d} \\
60 \mathrm{c} \\
100 \\
6 \\
6 \\
\\
\\
8 \\
10 \mathrm{~h} \\
40\end{array}$ & $\begin{array}{l}2853.554 \\
2853.643 \\
2855.637 \\
2857.128 \\
2858.482 \\
2859.110 \\
2859.852 \\
2860.770 \\
2860.988 \\
2861.361\end{array}$ & $\begin{array}{c}10 \\
500 \mathrm{~h} \\
10 \mathrm{~h} \\
2000 \mathrm{c} \\
\\
6 \mathrm{c} \\
15\end{array}$ & $\begin{array}{c}1 \\
2 \\
1 \\
7 \\
\\
200 \mathrm{c} \\
2 \\
600 \mathrm{c} \\
3 \mathrm{~h}\end{array}$ & $\begin{array}{l}2904.956 \\
2905.420 \\
2906.251 \\
2906.618 \\
2906.882 \\
2907.625 \\
2907.856 \\
2907.982 \\
2909.036 \\
2909.074\end{array}$ & $\begin{array}{r}15 \\
7\end{array}$ & $\begin{array}{r}1 \\
8 \\
100 \\
9 \mathrm{c} \\
10 \mathrm{c} \\
4\end{array}$ \\
\hline $\begin{array}{l}2778.908 \\
2779.773 \\
2781.222 \\
2782.052 \\
2782.235 \\
2782.955 \\
2784.372 \\
2784.726 \\
2785.134 \\
2785.288\end{array}$ & $\begin{array}{r}150 \\
1 \\
25 \\
1000\end{array}$ & $\begin{array}{c}9 \\
30 \\
\\
15 \\
3 \\
1 \\
10 \mathrm{~h} \\
5 \\
8 \\
20\end{array}$ & $\begin{array}{l}2817.286 \\
2817.612 \\
2818.630 \\
2819.050 \\
2819.460 \\
2820.198 \\
2820.645 \\
2821.352 \\
2821.590 \\
2822.910\end{array}$ & $\begin{array}{r}40 \\
\\
100 \\
5\end{array}$ & $\begin{array}{c}7 \\
15 \\
15 \\
4 \\
1 \\
20 \\
61 \\
500 \\
100 \mathrm{w} \\
8 \mathrm{~h}\end{array}$ & $\begin{array}{l}2861.684 \\
2862.772 \\
2863.335 \\
2863.528 \\
2864.486 \\
2864.892 \\
2866.069 \\
2867.213 \\
2867.430 \\
2867.673\end{array}$ & $\begin{array}{c}10 \\
500 \\
\\
8 \mathrm{c} \\
8 \\
8\end{array}$ & $\begin{array}{c}30 \\
4 \\
\\
4 \\
8 \\
6 \\
300 \mathrm{c}\end{array}$ & $\begin{array}{l}2909.508 \\
2909.852 \\
2910.202 \\
2910.689 \\
2911.702 \\
2911.996 \\
2913.147 \\
2913.792 \\
2914.242 \\
2915.394\end{array}$ & $\begin{array}{r}5 \\
10 w \\
\\
2 \\
1000 \\
8\end{array}$ & $\begin{array}{c}1 \\
150 \mathrm{w} \\
6 \\
6 \\
10 \\
7 \\
\\
3 \\
6\end{array}$ \\
\hline $\begin{array}{l}2785.586 \\
2786.941 \\
2787.234 \\
2787.609 \\
2787.937 \\
2788.797 \\
2788.888 \\
2789.246 \\
2789.273 \\
2790.293\end{array}$ & $\begin{array}{r}500 \\
15\end{array}$ & $\begin{array}{c}10 \\
1 \\
2 \\
2 \\
30 \\
7 \mathrm{~h}\end{array}$ & $\begin{array}{l}2824.380 \\
2825.042 \\
2825.342 \\
2825.967 \\
2826.166 \\
2828.042 \\
2829.090 \\
2829.375 \\
2829.607 \\
2830.202\end{array}$ & $\begin{array}{c}6 c \\
6 c \\
10 \\
2 c \\
200 \\
5\end{array}$ & $\begin{array}{r}3 \mathrm{~h} \\
80 \mathrm{c} \\
100 \mathrm{c} \\
\\
100 \mathrm{c} \\
10 \\
4 \\
10 \mathrm{~s} \\
1\end{array}$ & $\begin{array}{l}2868.087 \\
2868.788 \\
2869.283 \\
2869.303 \\
2869.497 \\
2870.295 \\
2870.422 \\
2871.438 \\
2871.986 \\
2872.234\end{array}$ & $\begin{array}{r}100 \\
4 \\
20 \\
\\
10\end{array}$ & $\begin{array}{c}3 \\
1 \\
\\
30 \\
1 \\
4 \\
15 \\
3 \mathrm{~h} \\
4 \\
30 \mathrm{cw}\end{array}$ & $\begin{array}{l}2915.850 \\
2919.650 \\
2920.850 \\
2921.054 \\
2921.496 \\
2921.912 \\
2922.736 \\
2923.155 \\
2923.342 \\
2924.254\end{array}$ & $\begin{array}{c}7 \\
15 \mathrm{w} \\
500 \\
3 \\
5 \\
20 \mathrm{c}\end{array}$ & $\begin{array}{c}3 \\
3 \\
\\
100 \mathrm{w} \\
50 \\
2 \\
70 \\
\\
200 \mathrm{c} \\
70\end{array}$ \\
\hline $\begin{array}{l}2790.443 \\
2790.628 \\
2790.920 \\
2791.739 \\
2792.070 \\
2792.973 \\
2793.672 \\
2794.228 \\
2794.526 \\
2795.072\end{array}$ & $\begin{array}{r}3 \\
100\end{array}$ & $\begin{array}{l}9 \\
1 \\
2 \mathrm{~h} \\
3 \\
7 \mathrm{c} \\
4 \\
4 \\
60 \\
1 \\
6\end{array}$ & $\begin{array}{l}2830.823 \\
2830.869 \\
2831.180 \\
2833.599 \\
2834.326 \\
2834.762 \\
2836.117 \\
2836.900 \\
2837.382 \\
2838.231\end{array}$ & $\begin{array}{r}4 \\
60\end{array}$ & $\begin{array}{c}3 \\
600 \\
200 \\
3 \\
2 \\
150 \\
3 \\
10 \mathrm{hw} \\
1\end{array}$ & $\begin{array}{l}2873.660 \\
2874.101 \\
2877.196 \\
2877.606 \\
2878.703 \\
2879.133 \\
2880.406 \\
2881.269 \\
2882.362 \\
2882.891\end{array}$ & $\begin{array}{l}1 \\
5\end{array}$ & $\begin{array}{c}10 \\
3 \\
1 \\
30 \\
50 \\
200 \mathrm{c} \\
20 \mathrm{hw} \\
60 \\
4\end{array}$ & $\begin{array}{l}2924.870 \\
2925.065 \\
2927.098 \\
2927.318 \\
2927.783 \\
2927.960 \\
2928.198 \\
2928.434 \\
2929.515 \\
2930.035\end{array}$ & $\begin{array}{r}1000 \\
1\end{array}$ & $\begin{array}{r}3 \\
7 \\
\\
10 \\
1 \\
1 \\
2 \\
40 \\
20 \\
18\end{array}$ \\
\hline
\end{tabular}


TABLE I. Spectrum of technetium - Continued

\begin{tabular}{|c|c|c|c|c|c|c|c|c|c|c|c|}
\hline \multirow{2}{*}{$\begin{array}{l}\text { Wave- } \\
\text { length }\end{array}$} & \multicolumn{2}{|c|}{ Intensity } & \multirow{2}{*}{$\begin{array}{l}\text { Wave- } \\
\text { length }\end{array}$} & \multicolumn{2}{|c|}{ Intensity } & \multirow{2}{*}{$\begin{array}{l}\text { Wave- } \\
\text { length }\end{array}$} & \multicolumn{2}{|c|}{ Intensity } & \multirow{2}{*}{$\begin{array}{l}\text { Wave- } \\
\text { length }\end{array}$} & \multicolumn{2}{|c|}{ Intensity } \\
\hline & Arc & Spark & & Arc & Spark & & Arc & Spark & & Arc & Spark \\
\hline $\begin{array}{l}2930.485 \\
2931.134 \\
2931.552 \\
2932.062 \\
2932.118 \\
2932.923 \\
2932.975 \\
2933.888 \\
2933.924 \\
2936.921\end{array}$ & $\begin{array}{r}4 \\
3 \\
80 \\
3\end{array}$ & $\begin{array}{r}1 \\
20 \\
6 \\
4 \\
1 \\
\\
\\
50 \\
50\end{array}$ & $\begin{array}{l}2978.254 \\
2979.338 \\
2979.752 \\
2979.780 \\
2980.832 \\
2982.217 \\
2982.552 \\
2983.712 \\
2984.137 \\
2984.362\end{array}$ & $\begin{array}{r}8 \\
20 \\
30\end{array}$ & $\begin{array}{c}2 \\
3 \\
7 \\
2 \mathrm{~h} \\
3 \mathrm{~h} \\
400 \\
20 \mathrm{~h} \\
1\end{array}$ & $\begin{array}{l}3026.445 \\
3026.887 \\
3029.220 \\
3030.276 \\
3030.718 \\
3031.220 \\
3031.486 \\
3032.089 \\
3033.158 \\
3033.621\end{array}$ & $\begin{array}{c}300 \mathrm{w} \\
6 \\
2\end{array}$ & $\begin{array}{c}4 \\
200 w \\
3 \\
\\
3 w \\
1 \\
10 \\
2 \\
4\end{array}$ & \begin{tabular}{|l}
3074.270 \\
3076.238 \\
3076.528 \\
3076.855 \\
3077.591 \\
3077.701 \\
3078.630 \\
3079.907 \\
3081.075 \\
3082.098
\end{tabular} & $\begin{array}{r}20 \\
80 \\
10 \\
12 \\
3\end{array}$ & $\begin{array}{c}100 \\
3 \mathrm{~h} \\
10 \\
1 \\
2 \mathrm{~h}\end{array}$ \\
\hline $\begin{array}{l}2937.801 \\
2938.868 \\
2938.993 \\
2939.501 \\
2939.990 \\
2940.487 \\
2940.928 \\
2941.835 \\
2942.082 \\
2942.912\end{array}$ & $\begin{array}{r}6 \\
10 \\
1 \\
15\end{array}$ & $\begin{array}{c}70 \\
1 \\
1 \\
2 \\
3 \\
8 \\
1 \\
100 \mathrm{c}\end{array}$ & $\begin{array}{l}2984.388 \\
2985.046 \\
2985.364 \\
2985.601 \\
2985.932 \\
2986.453 \\
2987.235 \\
2988.172 \\
2988.468 \\
2988.614\end{array}$ & $\begin{array}{r}15 \\
150 \\
8 \\
8 \\
15 \\
\\
20 \mathrm{c} \\
5\end{array}$ & $\begin{array}{r}3 \\
10\end{array}$ & $\begin{array}{l}3034.568 \\
3034.642 \\
3034.963 \\
3035.512 \\
3036.014 \\
3036.878 \\
3037.449 \\
3037.902 \\
3038.226 \\
3040.228\end{array}$ & $\begin{array}{r}80 \\
\\
\\
40 \\
3 \\
20 \\
100\end{array}$ & $\begin{array}{r}3 \\
2 \\
1 \\
7 \\
2 \\
\\
800 \\
2 \\
3\end{array}$ & $\begin{array}{l}3082.886 \\
3083.289 \\
3083.354 \\
3084.499 \\
3085.752 \\
3085.906 \\
3089.120 \\
3089.340 \\
3090.222 \\
3092.517\end{array}$ & $\begin{array}{r}3 w \\
20 \\
12 \\
150 \\
30\end{array}$ & $\begin{array}{c}3 \mathrm{~h} \\
15 \mathrm{c} \\
\\
4 \mathrm{~h} \\
1 \\
\\
3 \\
2 \\
1 \\
2 \mathrm{~h}\end{array}$ \\
\hline $\begin{array}{l}2945.518 \\
2946.220 \\
2946.581 \\
2947.107 \\
2948.070 \\
2948.473 \\
2948.633 \\
2949.255 \\
2949.456 \\
2949.580\end{array}$ & $\begin{array}{r}15 \\
\\
8 \\
15 \\
20 \\
\\
25\end{array}$ & $\begin{array}{c}20 \\
2 \\
8 \\
1 \\
3 \\
100 \mathrm{c} \\
400 \mathrm{c} \\
\\
10 \mathrm{~h} \\
1\end{array}$ & $\begin{array}{l}2990.132 \\
2990.748 \\
2992.873 \\
2993.232 \\
2993.460 \\
2993.762 \\
2993.910 \\
2994.097 \\
2996.882 \\
2997.076\end{array}$ & $\begin{array}{c}8 \mathrm{w} \\
\\
30 \mathrm{~h} \\
18 \mathrm{~h} \\
8 \\
\\
1 \\
15 \\
20\end{array}$ & $\begin{array}{r}15 \\
1 \\
8 \\
4\end{array}$ & $\begin{array}{l}3040.605 \\
3040.874 \\
3042.213 \\
3042.359 \\
3042.643 \\
3043.049 \\
3043.774 \\
3043.938 \\
3044.686 \\
3044.978\end{array}$ & $\begin{array}{r}15 \\
12 \\
\\
40 \\
12 \\
8 \\
8 \\
\\
10\end{array}$ & $\begin{array}{l}1 \\
1 \\
2 \\
3 \\
2 \\
1\end{array}$ & $\begin{array}{l}3092.749 \\
3093.617 \\
3093.988 \\
3095.014 \\
3095.740 \\
3095.834 \\
3096.672 \\
3096.710 \\
3097.269 \\
3097.582\end{array}$ & $\begin{array}{l}20 \mathrm{w} \\
30 \\
5 \\
\\
5\end{array}$ & $\begin{array}{c}30 \mathrm{c} \\
10 \\
1\end{array}$ \\
\hline $\begin{array}{l}2950.248 \\
2950.375 \\
2950.803 \\
2951.033 \\
2952.068 \\
2952.544 \\
2953.162 \\
2953.838 \\
2954.046 \\
2954.149\end{array}$ & $\begin{array}{l}8 \mathrm{~h} \\
10 \mathrm{~h} \\
10 \mathrm{~h}\end{array}$ & $\begin{array}{r}15 \\
50 \\
30 \\
10 \\
20 \\
40 \\
4\end{array}$ & $\begin{array}{l}2997.186 \\
2997.635 \\
2997.906 \\
2997.953 \\
2998.235 \\
2998.356 \\
2999.690 \\
3000.418 \\
3001.055 \\
3001.590\end{array}$ & $\begin{array}{r}1 \\
20 \\
6\end{array}$ & $\begin{array}{c}8 \\
\\
2 \\
8 \mathrm{~h} \\
2 \\
1 \\
150 \\
2\end{array}$ & $\begin{array}{l}3045.292 \\
3046.502 \\
3047.113 \\
3047.529 \\
3049.047 \\
3050.818 \\
3051.332 \\
3051.550 \\
3051.600 \\
3052.128\end{array}$ & $\begin{array}{c}4 \\
\\
6 \\
4 \\
100 \mathrm{~h} \\
\\
20 \mathrm{w}\end{array}$ & $\begin{array}{r}10 \mathrm{c} \\
20 \mathrm{c} \\
15 \mathrm{~h} \\
6 \mathrm{~h}\end{array}$ & $\begin{array}{l}3098.556 \\
3098.657 \\
3099.098 \\
3099.517 \\
3100.462 \\
3100.638 \\
3100.880 \\
3101.582 \\
3101.782 \\
3102.822\end{array}$ & $\begin{array}{r}20 \\
1000 \\
200\end{array}$ & $\begin{array}{r}40 \\
10 \\
2 \\
4 \\
3 \\
30 \\
\\
2\end{array}$ \\
\hline $\begin{array}{l}2955.928 \\
2956.229 \\
2957.823 \\
2958.357 \\
2958.424 \\
2958.547 \\
2958.832 \\
2961.636 \\
2962.086 \\
2962.569\end{array}$ & $\begin{array}{r}200 \\
6 \\
3 \\
\\
2 \\
1 \\
7\end{array}$ & $\begin{array}{r}3 \\
3 \\
1 \\
\\
7 \\
\\
5 \\
30 \\
40 \\
4\end{array}$ & $\begin{array}{l}3002.269 \\
3002.669 \\
3003.022 \\
3003.698 \\
3004.379 \\
3004.601 \\
3005.488 \\
3007.007 \\
3008.782 \\
3009.746\end{array}$ & $\begin{array}{l}10 \\
15 \\
\\
30\end{array}$ & $\begin{array}{c}5 \\
10 \\
1 \\
15 \\
5 \\
9 \mathrm{w} \\
100 \mathrm{c} \\
2 \\
6\end{array}$ & $\begin{array}{l}3052.474 \\
3052.507 \\
3053.106 \\
3055.464 \\
3056.670 \\
3057.014 \\
3057.327 \\
3057.515 \\
3058.509 \\
3059.308\end{array}$ & $\begin{array}{r}40 \\
10 \\
5 \\
5 \\
30 \\
30\end{array}$ & $\begin{array}{c}10 \mathrm{w} \\
7 \\
5 \\
\\
4 \\
2 \\
10 \\
20 \\
1\end{array}$ & $\begin{array}{l}3103.443 \\
3104.293 \\
3105.107 \\
3105.387 \\
3106.937 \\
3108.254 \\
3108.576 \\
3109.122 \\
3109.151 \\
3110.563\end{array}$ & $\begin{array}{l}10 \\
30\end{array}$ & $\begin{array}{c}1 \\
70 \\
10 \\
2 \\
2 \\
1 \\
20 \mathrm{c} \\
\\
30\end{array}$ \\
\hline $\begin{array}{l}2964.287 \\
2964.487 \\
2965.488 \\
2966.196 \\
2966.550 \\
2967.084 \\
2967.242 \\
2967.956 \\
2968.233 \\
2968.769\end{array}$ & $\begin{array}{l}20 \\
10\end{array}$ & $\begin{array}{c}800 \\
3 \\
50 \\
15 \\
\\
10 \mathrm{~h} \mathrm{l} \\
2 \mathrm{~h} \\
1\end{array}$ & $\begin{array}{l}3010.831 \\
3011.868 \\
3012.388 \\
3015.161 \\
3016.298 \\
3017.232 \\
3018.341 \\
3019.179 \\
3020.452 \\
3021.555\end{array}$ & $\begin{array}{r}100 \\
\\
2 \\
12 \\
300 \\
4 \\
3 \\
3 \\
150\end{array}$ & $\begin{array}{c}2 \\
1 \mathrm{~h} \\
1 \mathrm{~h} \\
30 \\
\\
7 \\
15 \\
3 \\
2\end{array}$ & $\begin{array}{l}3059.550 \\
3060.103 \\
3060.672 \\
3060.904 \\
3061.283 \\
3061.780 \\
3062.112 \\
3062.361 \\
3064.667 \\
3066.393\end{array}$ & $\begin{array}{r}6 \\
4 \\
30 \\
\\
3 \\
\\
80 \\
200 \\
300\end{array}$ & $\begin{array}{r}1 \\
4 \\
\\
10 \\
2 \\
2 \\
3 \\
4\end{array}$ & $\begin{array}{l}3110.759 \\
3110.938 \\
3111.392 \\
3115.296 \\
3115.977 \\
3116.382 \\
3116.698 \\
3116.914 \\
3118.644 \\
3119.171\end{array}$ & $\begin{array}{c}15 \\
3 \\
10 w \\
\\
60 \\
\\
2 \\
10 \\
5 \\
80\end{array}$ & $\begin{array}{l}4 \\
2 \\
3\end{array}$ \\
\hline $\begin{array}{l}2969.770 \\
2970.186 \\
2970.740 \\
2970.760 \\
2970.900 \\
2973.179 \\
2973.650 \\
2976.179 \\
2976.379 \\
2976.561\end{array}$ & $\begin{array}{r}20 \\
18 \\
200 \\
\\
5 \\
15 \mathrm{c}\end{array}$ & $\begin{array}{l}2 \\
40 \mathrm{~h} 1 \\
20 \mathrm{~h}\end{array}$ & $\begin{array}{l}3022.035 \\
3022.203 \\
3022.578 \\
3022.665 \\
3022.998 \\
3023.680 \\
3024.208 \\
3025.263 \\
3025.523 \\
3025.932\end{array}$ & $\begin{array}{r}100 \\
200 \\
80 \\
3\end{array}$ & $\begin{array}{c}20 \mathrm{~h} \\
10 \mathrm{~h} \\
\\
1 \\
3 \\
1 \\
3 \\
1\end{array}$ & $\begin{array}{l}3066.602 \\
3068.337 \\
3069.956 \\
3070.241 \\
3072.702 \\
3072.898 \\
3073.046 \\
3073.326 \\
3073.726 \\
3073.969\end{array}$ & $\begin{array}{c}100 \mathrm{c} \\
120 \mathrm{c} \\
3 \\
30 \\
8 \\
\\
10 \\
12\end{array}$ & 200 & $\begin{array}{l}3119.662 \\
3119.888 \\
3120.937 \\
3121.306 \\
3121.970 \\
3122.642 \\
3123.556 \\
3126.184 \\
3126.390 \\
3126.807\end{array}$ & $\begin{array}{c}40 \\
1 \\
10 \mathrm{~h} \\
700 \\
10 \\
8 \mathrm{~W} \\
5\end{array}$ & $\begin{array}{c}1 \\
40 \\
2 \mathrm{~h} \\
50 \\
3 \\
5\end{array}$ \\
\hline
\end{tabular}


TABLE I. Spectrum of technetium - Continued

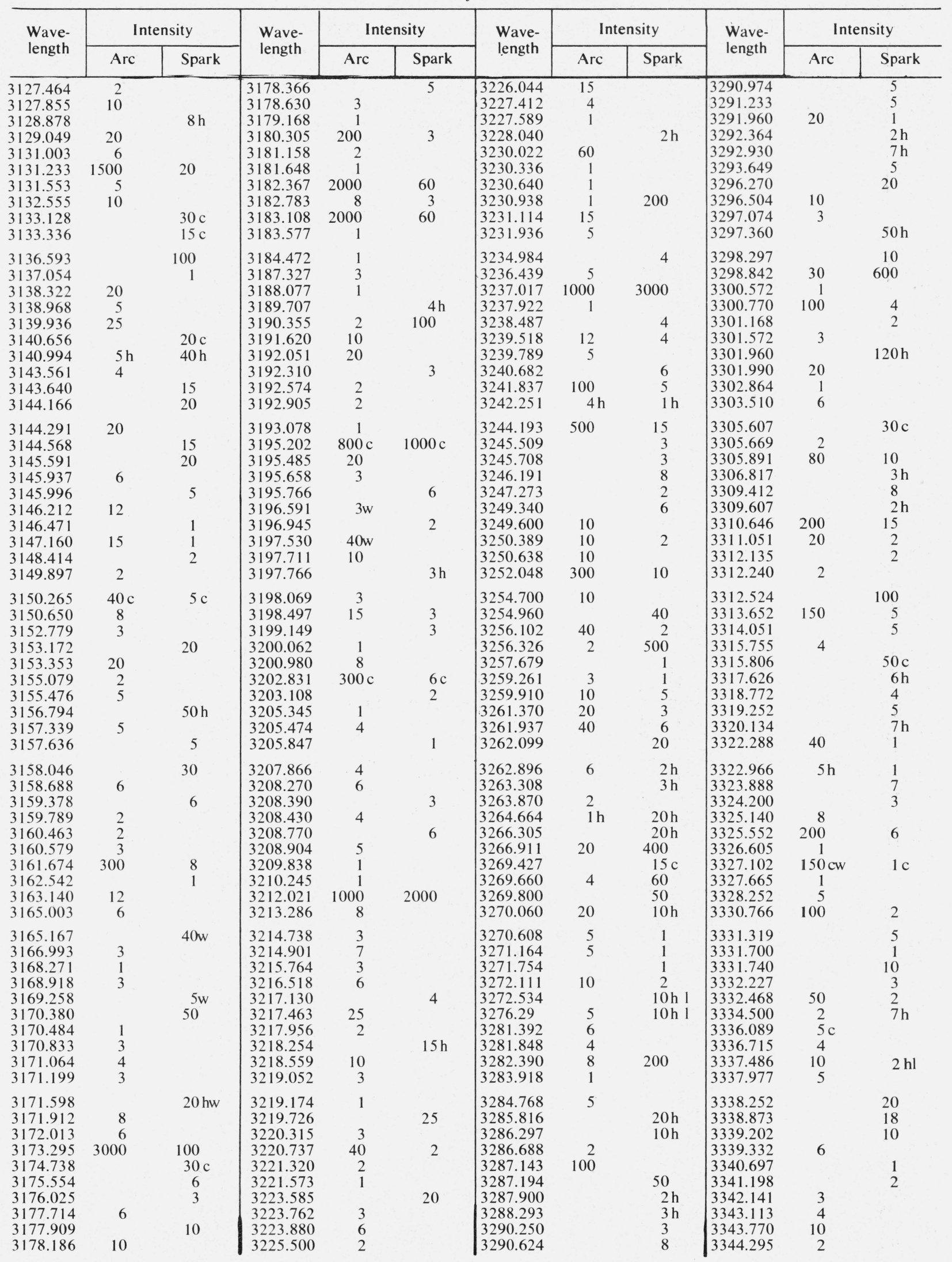


TABLE I. Spectrum of technetium - Continued

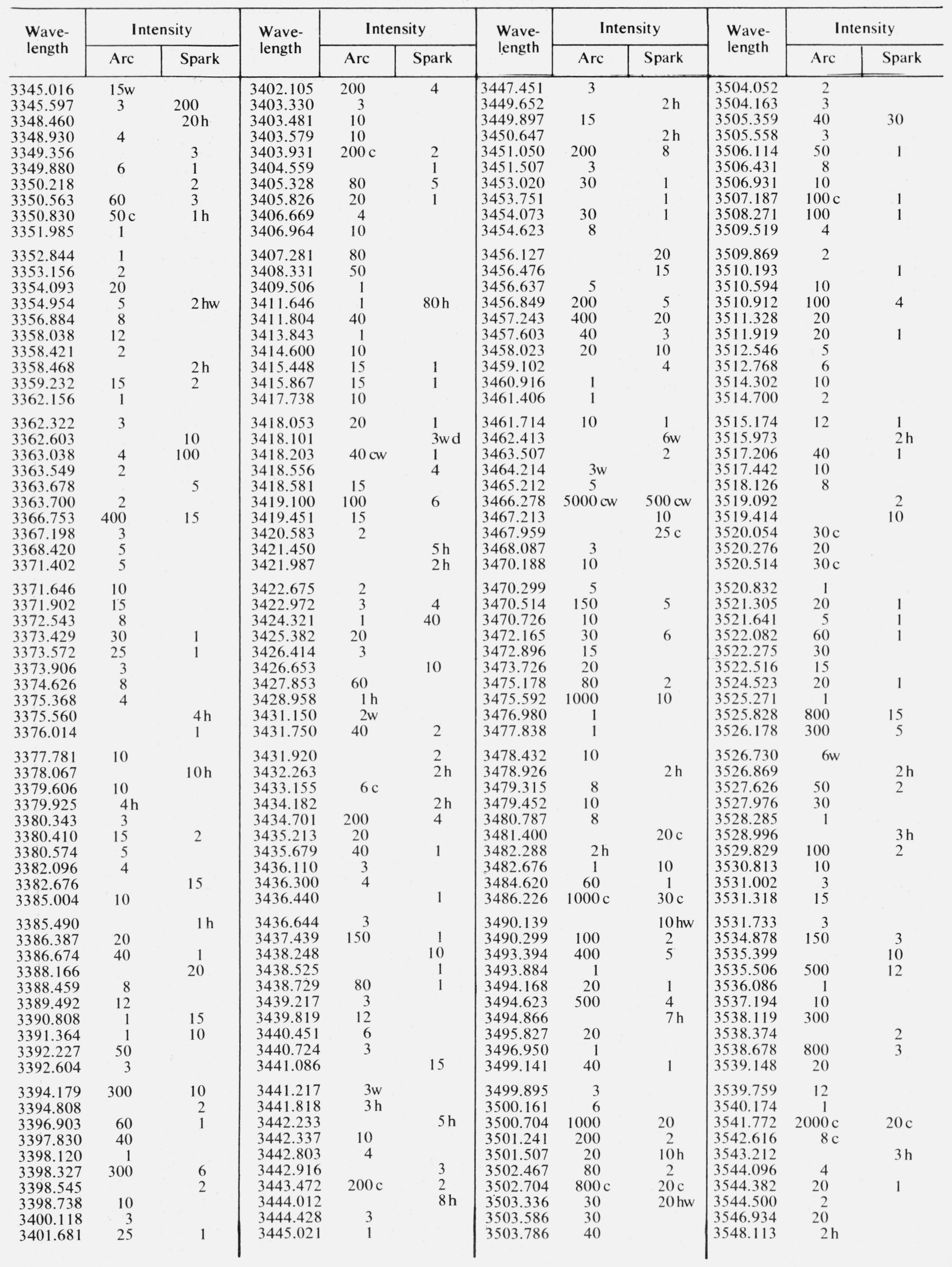


TABLE I. Spectrum of technetium - Continued

\begin{tabular}{|c|c|c|c|c|c|c|c|c|c|c|c|}
\hline \multirow{2}{*}{$\begin{array}{l}\text { Wave- } \\
\text { length }\end{array}$} & \multicolumn{2}{|c|}{ Intensity } & \multirow{2}{*}{$\begin{array}{l}\text { Wave- } \\
\text { length }\end{array}$} & \multicolumn{2}{|c|}{ Intensity } & \multirow{2}{*}{$\begin{array}{l}\text { Wave- } \\
\text { length }\end{array}$} & \multicolumn{2}{|c|}{ Intensity } & \multirow{2}{*}{$\begin{array}{l}\text { Wave- } \\
\text { length }\end{array}$} & \multicolumn{2}{|c|}{ Intensity } \\
\hline & Arc & Spark & & Arc & Spark & & Arc & Spark & & Arc & Spark \\
\hline $\begin{array}{l}3548.825 \\
3549.725 \\
3550.645 \\
3551.206 \\
3551.998 \\
3552.828 \\
3553.539 \\
3554.580 \\
3554.919 \\
3556.056\end{array}$ & $\begin{array}{r}2 \mathrm{~h} \\
6000 \mathrm{c} \\
4000 \mathrm{c} \\
30 \\
3 \\
4 \\
60 \\
30 \\
2 \\
40\end{array}$ & $\begin{array}{l}60 c \\
40 c\end{array}$ & $\begin{array}{l}3595.661 \\
3596.181 \\
3597.401 \\
3598.261 \\
3598.830 \\
3599.152 \\
3599.750 \\
3602.191 \\
3602.639 \\
3604.794\end{array}$ & $\begin{array}{c}1000 \mathrm{cW} \\
4 \\
12 \\
30 \\
\\
50 \\
20 \\
1 \\
1\end{array}$ & $\begin{array}{l}10 \mathrm{cw} \\
1 \\
2 \mathrm{~h} \\
1 \\
1 \\
3 \mathrm{~h}\end{array}$ & $\begin{array}{l}3643.950 \\
3644.134 \\
3644.427 \\
3645.630 \\
3645.796 \\
3646.851 \\
3648.040 \\
3648.860 \\
3650.344 \\
3651.467\end{array}$ & $\begin{array}{c}8 \\
50 \\
3 \\
2 \\
2 \\
1000 \mathrm{c} \\
10 \\
60 \\
600\end{array}$ & $\begin{array}{c}2 \\
50 \mathrm{c} \\
2 \\
20\end{array}$ & $\begin{array}{l}3702.366 \\
3702.498 \\
3703.176 \\
3703.830 \\
3704.797 \\
3705.481 \\
3705.712 \\
3706.705 \\
3707.627 \\
3708.261\end{array}$ & $\begin{array}{r}8 \\
\\
60 \\
800 \\
300 \\
100 \\
80 \\
200 \\
200 \\
200\end{array}$ & $\begin{array}{c}2 \mathrm{~h} \\
2 \\
20 \\
5 \\
2 \\
1 \\
4 \\
5 \\
3\end{array}$ \\
\hline $\begin{array}{l}3556.178 \\
3558.209 \\
3558.260 \\
3558.662 \\
3559.111 \\
3559.390 \\
3559.754 \\
3560.319 \\
3560.876 \\
3561.192\end{array}$ & $\begin{array}{c}15 \\
3 \\
1 \mathrm{~h} \\
300 \\
800 \\
50 \\
50\end{array}$ & $\begin{array}{l}1 \\
1 \mathrm{~h} \\
\\
1 \mathrm{~h} \\
1 \mathrm{~h} \\
5 \\
15 \\
1\end{array}$ & $\begin{array}{l}3605.717 \\
3605.938 \\
3606.265 \\
3607.321 \\
3607.625 \\
3608.271 \\
3609.045 \\
3609.527 \\
3609.843 \\
3609.906\end{array}$ & $\begin{array}{c}7 \\
15 \\
\\
1000 \mathrm{c} \\
200 \\
2000 \mathrm{c} \\
60 \\
1 \\
6 \\
5\end{array}$ & $\begin{array}{c}2 \mathrm{~h} \\
30 \mathrm{c} \\
5 \\
80 \mathrm{c}\end{array}$ & $\begin{array}{l}3654.054 \\
3656.458 \\
3658.152 \\
3658.588 \\
3659.882 \\
3661.452 \\
3662.032 \\
3662.838 \\
3663.091 \\
3664.455\end{array}$ & $\begin{array}{c}10 \\
1 \\
10 \\
1000 \mathrm{c} \\
3 \\
400 \mathrm{c} \\
6 \\
30 \\
8 \\
6\end{array}$ & $\begin{array}{c}60 c \\
30 c \\
1\end{array}$ & $\begin{array}{l}3709.226 \\
3709.527 \\
3709.831 \\
3710.126 \\
3710.428 \\
3711.048 \\
3711.388 \\
3712.256 \\
3712.819 \\
3713.838\end{array}$ & $\begin{array}{r}2 \\
15 \\
80 \\
90 \\
4 \\
2 \\
80 \\
1000 \\
300 \\
25\end{array}$ & $\begin{array}{r}1 \\
10 \\
3\end{array}$ \\
\hline $\begin{array}{l}3562.303 \\
3563.000 \\
3563.994 \\
3564.359 \\
3564.566 \\
3565.008 \\
3565.217 \\
3565.490 \\
3566.883 \\
3568.853\end{array}$ & $\begin{array}{r}4 \\
5 \\
5 \\
1 \\
\\
100 \\
80 \\
3 \\
800\end{array}$ & $\begin{array}{l}3 \\
1 \\
1 \\
\\
5\end{array}$ & $\begin{array}{l}3610.354 \\
3610.550 \\
3611.109 \\
3611.156 \\
3611.788 \\
3612.108 \\
3612.346 \\
3612.833 \\
3613.163 \\
3613.232\end{array}$ & $\begin{array}{c}1 \\
10 \mathrm{c} \\
2 \\
50 \\
4 \\
60 \\
3 \\
4 \\
\\
3\end{array}$ & $\begin{array}{l}3 \mathrm{~h} \\
1 \\
1\end{array}$ & $\begin{array}{l}3664.575 \\
3664.916 \\
3667.349 \\
3667.510 \\
3667.794 \\
3667.991 \\
3668.940 \\
3669.152 \\
3669.918 \\
3671.710\end{array}$ & $\begin{array}{r}200 \\
10 \\
30 \\
10 \\
10 \\
20 \\
40 \\
40\end{array}$ & $\begin{array}{l}7 \mathrm{~h} \\
2 \\
1 \\
\\
1 \\
2 \\
1 \mathrm{~h} \\
2\end{array}$ & $\begin{array}{l}3714.516 \\
3715.944 \\
3716.706 \\
3716.809 \\
3717.024 \\
3717.287 \\
3718.861 \\
3720.407 \\
3720.688 \\
3721.056\end{array}$ & $\begin{array}{r}100 \\
500 \\
4 \\
20 \\
15 \\
1 \\
10000\end{array}$ & $\begin{array}{c}1 \\
5 \\
\\
2 \\
1 \\
8 \\
300 \\
2 \mathrm{~h} \\
2 \mathrm{~h}\end{array}$ \\
\hline $\begin{array}{l}3569.852 \\
3570.654 \\
3572.631 \\
3573.083 \\
3573.306 \\
3573.654 \\
3574.410 \\
3574.610 \\
3575.420 \\
3575.463\end{array}$ & $\begin{array}{c}30 \mathrm{~s} \\
100 \\
1 \\
50 \\
10 \mathrm{~h} \\
1 \\
25 \\
80 \\
100\end{array}$ & $\begin{array}{c}1 \\
10 \\
4 \mathrm{~h}\end{array}$ & $\begin{array}{l}3614.282 \\
3615.814 \\
3616.346 \\
3616.528 \\
3617.692 \\
3618.582 \\
3618.943 \\
3619.664 \\
3619.795 \\
3622.453\end{array}$ & $\begin{array}{c}15 \\
50 \\
\\
30 \\
20 \\
8 \mathrm{~h} \\
200 \\
20 \\
10 \\
1\end{array}$ & $10 \mathrm{~h}$ & $\begin{array}{l}3672.087 \\
3672.553 \\
3673.014 \\
3673.285 \\
3675.003 \\
3675.429 \\
3675.584 \\
3675.855 \\
3675.899 \\
3678.089\end{array}$ & $\begin{array}{r}8 \\
3 \\
1 \\
3 \\
30 \\
10 \mathrm{w} \\
80\end{array}$ & $\begin{array}{l}4 \mathrm{~h} \\
5 \\
4 \mathrm{hw} \\
2\end{array}$ & $\begin{array}{l}3721.160 \\
3722.885 \\
3723.132 \\
3723.674 \\
3724.176 \\
3724.395 \\
3725.082 \\
3726.152 \\
3726.351 \\
3727.364\end{array}$ & $\begin{array}{r}40 \\
1500 \\
10 \\
2000 \\
20 \\
100 \\
5000 \\
200\end{array}$ & 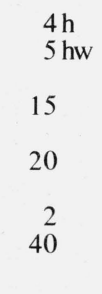 \\
\hline $\begin{array}{l}3575.964 \\
3576.324 \\
3576.495 \\
3577.228 \\
3577.796 \\
3577.986 \\
3578.764 \\
3578.969 \\
3580.059 \\
3581.258\end{array}$ & $\begin{array}{r}20 \\
50 \\
\\
6 \\
1 \\
15 \\
12 \\
60 \\
1000 \\
600\end{array}$ & $\begin{array}{c}10 \mathrm{c} \\
3\end{array}$ & $\begin{array}{l}3623.508 \\
3625.555 \\
3626.294 \\
3627.364 \\
3627.858 \\
3628.476 \\
3628.901 \\
3630.388 \\
3631.227 \\
3633.244\end{array}$ & $\begin{array}{c}5 \\
150 \\
10 \\
1000 \mathrm{c} \\
100 \\
2 \\
30 \\
200 \\
2 \\
150\end{array}$ & $\begin{array}{c}3 \\
10 c \\
2 \\
1 \\
2 \\
2\end{array}$ & $\begin{array}{l}3678.388 \\
3679.146 \\
3679.603 \\
3680.318 \\
3680.518 \\
3680.575 \\
3681.683 \\
3682.592 \\
3683.073 \\
3684.744\end{array}$ & $\begin{array}{r}70 \\
1000 \\
1 \\
300 \\
\\
50 \\
40 \\
100 \\
2 \\
5000\end{array}$ & $\begin{array}{l}2 \\
30 \\
\\
6 \\
8 \mathrm{~h}\end{array}$ & $\begin{array}{l}3728.291 \\
3728.886 \\
3729.177 \\
3729.618 \\
3730.358 \\
3730.836 \\
3731.412 \\
3731.735 \\
3735.501 \\
3735.753\end{array}$ & $\begin{array}{c}2 \\
30 \\
400 \mathrm{c} \\
2 \\
10 \\
60 \\
8 \\
500 \\
30 \\
4\end{array}$ & $\begin{array}{l}1 \\
3\end{array}$ \\
\hline $\begin{array}{l}3581.744 \\
3582.075 \\
3582.628 \\
3583.464 \\
3584.005 \\
3585.684 \\
3586.566 \\
3587.386 \\
3587.942 \\
3589.471\end{array}$ & $\begin{array}{r}10 \\
800 \\
2000 \\
12 \\
1 \\
30 \\
3 \\
1 \\
4000 \\
15\end{array}$ & $\begin{array}{r}4 \\
10\end{array}$ & $\begin{array}{l}3634.258 \\
3634.764 \\
3634.800 \\
3635.146 \\
3636.070 \\
3637.447 \\
3638.012 \\
3638.220 \\
3638.848 \\
3639.069\end{array}$ & $\begin{array}{c}1 \mathrm{~h} \\
10 \\
3000 \mathrm{c} \\
10000 \mathrm{c} \\
8 \\
1 \\
1000 \\
200 \\
1\end{array}$ & $\begin{array}{c}8 \\
3 h \\
50 c \\
500 c\end{array}$ & $\begin{array}{l}3686.027 \\
3687.772 \\
3691.324 \\
3691.608 \\
3691.674 \\
3692.759 \\
3693.400 \\
3694.327 \\
3695.404 \\
3695.734\end{array}$ & $\begin{array}{c}20 \\
1 \\
50 \\
300 \\
8 \\
30 \mathrm{~d} \\
1\end{array}$ & $\begin{array}{l}1 \mathrm{~h} \\
1 \\
1 \mathrm{~h} \\
5 \mathrm{c} \\
4 \\
1 \\
1\end{array}$ & $\begin{array}{l}3737.420 \\
3737.662 \\
3737.693 \\
3738.501 \\
3739.342 \\
3740.388 \\
3740.494 \\
3740.988 \\
3742.789 \\
3743.508\end{array}$ & $\begin{array}{c}300 \\
10 \mathrm{~h} \\
20 \\
1 \\
1 \\
1 \\
2 \\
150 \\
1\end{array}$ & $\begin{array}{c}2 \\
20 \mathrm{~h}\end{array}$ \\
\hline $\begin{array}{l}3589.890 \\
3590.471 \\
3591.222 \\
3591.483 \\
3591.942 \\
3592.920 \\
3593.471 \\
3593.580 \\
3594.573 \\
3594.908\end{array}$ & $\begin{array}{c}3 \mathrm{~h} \\
1 \\
50 \\
80 \\
8 \mathrm{cw} \\
\\
200 \\
10 \\
300 \\
50 \mathrm{cw}\end{array}$ & $\begin{array}{l}2 \mathrm{~h} \\
1 \\
4 \\
1 \mathrm{~h} \\
3 \\
4\end{array}$ & $\begin{array}{l}3639.379 \\
3640.095 \\
3640.226 \\
3641.093 \\
3641.183 \\
3641.534 \\
3642.576 \\
3642.985 \\
3643.157 \\
3643.376\end{array}$ & $\begin{array}{r}900 \\
400 \\
6 \\
15 \\
30 \\
\\
30 \\
3 \\
10\end{array}$ & $\begin{array}{l}15 \\
10 \mathrm{w} \\
10 \\
1 \\
1 \\
1 \\
2 \\
1\end{array}$ & $\begin{array}{l}3695.999 \\
3696.297 \\
3696.747 \\
3697.415 \\
3697.736 \\
3698.694 \\
3698.959 \\
3701.478 \\
3701.966 \\
3702.077\end{array}$ & $\begin{array}{c}30 \mathrm{w} \\
150 \\
\\
100 \\
30 \\
\\
3 \\
5 \\
1\end{array}$ & $\begin{array}{l}1 \mathrm{~h} \\
3 \\
2 \mathrm{~h} \\
1 \\
1 \\
3 \mathrm{hw} \\
1 \\
3 \mathrm{~h}\end{array}$ & $\begin{array}{l}3743.610 \\
3744.026 \\
3744.801 \\
3745.012 \\
3745.808 \\
3746.148 \\
3746.640 \\
3746.845 \\
3748.406 \\
3749.634\end{array}$ & $\begin{array}{c}2 \\
150 \\
400 \\
4 \mathrm{hl} \\
1000 \\
100 \\
5000 \\
1 \\
10\end{array}$ & $\begin{array}{c}2 \\
6 \mathrm{~h} \\
5 \\
\\
10 \\
2 \\
150\end{array}$ \\
\hline
\end{tabular}


TABLE I. Spectrum of technetium $\rightarrow$ Continued

\begin{tabular}{|c|c|c|c|c|c|c|c|c|c|c|c|}
\hline \multirow{2}{*}{$\begin{array}{l}\text { Wave- } \\
\text { length }\end{array}$} & \multicolumn{2}{|c|}{ Intensity } & \multirow{2}{*}{$\begin{array}{l}\text { Wave- } \\
\text { length }\end{array}$} & \multicolumn{2}{|c|}{ Intensity } & \multirow{2}{*}{$\begin{array}{l}\text { Wave- } \\
\text { length }\end{array}$} & \multicolumn{2}{|c|}{ Intensity } & \multirow{2}{*}{$\begin{array}{l}\text { Wave- } \\
\text { length }\end{array}$} & \multicolumn{2}{|c|}{ Intensity } \\
\hline & Arc & Spark & & Arc & Spark & & Arc & Spark & & Arc & Spark \\
\hline $\begin{array}{l}3749.928 \\
3750.061 \\
3750.566 \\
3751.056 \\
3752.024 \\
3752.134 \\
3752.944 \\
3754.048 \\
3754.368 \\
3754.885\end{array}$ & $\begin{array}{r}60 \\
40 \\
6 \\
6 \\
30 \\
1000 \\
20 \\
60 \\
4000 \\
4\end{array}$ & $\begin{array}{r}1 \\
10 \\
\\
3 \\
40\end{array}$ & $\begin{array}{l}3791.524 \\
3791.734 \\
3792.102 \\
3792.674 \\
3792.774 \\
3793.087 \\
3793.207 \\
3793.721 \\
3793.986 \\
3794.800\end{array}$ & $\begin{array}{r}3 \\
300 \\
20 \\
6 \\
1 \\
3 \\
1 \\
2 \\
3 \mathrm{c} \\
60\end{array}$ & 1 & $\begin{array}{l}3847.351 \\
3847.596 \\
3847.870 \\
3848.310 \\
3849.519 \\
3850.553 \\
3851.222 \\
3852.328 \\
3853.323 \\
3854.074\end{array}$ & $\begin{array}{c}100 \\
500 \\
10 \mathrm{~h} \\
5 \mathrm{~h} \\
1 \\
5 \\
300 \\
80 \\
2 \\
8\end{array}$ & $\begin{array}{l}1 \\
3 \\
1\end{array}$ & \begin{tabular}{|l}
3908.596 \\
3909.038 \\
3909.782 \\
3909.958 \\
3911.157 \\
3911.921 \\
3914.538 \\
3914.723 \\
3915.174 \\
3915.889
\end{tabular} & $\begin{array}{r}20 \\
8 \\
20 \\
10 \\
5 \\
3 \\
3 \\
1 \\
2 \\
10\end{array}$ & \\
\hline $\begin{array}{l}3755.048 \\
3755.221 \\
3755.652 \\
3756.230 \\
3756.334 \\
3757.722 \\
3758.535 \\
3758.948 \\
3759.170 \\
3759.305\end{array}$ & $\begin{array}{c}3 \\
80 \\
10 \mathrm{c} \\
12 \\
\\
3 \\
1000 \\
5 \\
5\end{array}$ & $\begin{array}{c}2 \mathrm{~h} \\
10 \\
7\end{array}$ & $\begin{array}{l}3795.502 \\
3796.522 \\
3797.435 \\
3797.767 \\
3798.055 \\
3798.981 \\
3799.692 \\
3801.645 \\
3802.025 \\
3803.976\end{array}$ & $\begin{array}{c}12 \\
200 \\
1000 \\
40 \\
20 \mathrm{c} \\
80 \\
\\
2 \mathrm{~h} \\
40\end{array}$ & $\begin{array}{l}4 \mathrm{~h} \\
5 \\
10 \\
1 \\
2 \\
5 \mathrm{hw} \\
1\end{array}$ & $\begin{array}{l}3854.326 \\
3854.768 \\
3855.555 \\
3855.914 \\
3856.543 \\
3856.734 \\
3857.388 \\
3858.063 \\
3860.129 \\
3861.259\end{array}$ & $\begin{array}{c}50 \\
10 \\
20 \\
1 \\
20 \\
500 \mathrm{c} \\
20 \\
\\
8 \\
25 \mathrm{c}\end{array}$ & $\begin{array}{l}5 \mathrm{~h} \\
4\end{array}$ & $\begin{array}{l}3916.536 \\
3916.941 \\
3917.443 \\
3917.743 \\
3919.148 \\
3919.375 \\
3920.550 \\
3922.134 \\
3923.663 \\
3924.131\end{array}$ & $\begin{array}{c}60 \\
5 \\
10 \mathrm{c} \\
\\
50 \\
300 \\
30 \mathrm{cw} \\
100 \\
300 \mathrm{c} \\
20\end{array}$ & $\begin{array}{l}4 \mathrm{~h} \\
3 \\
1 \\
3 \mathrm{c}\end{array}$ \\
\hline $\begin{array}{l}3759.553 \\
3761.481 \\
3761.807 \\
3762.880 \\
3763.974 \\
3764.566 \\
3765.576 \\
3765.962 \\
3767.858 \\
3768.365\end{array}$ & $\begin{array}{r}20 \\
8 \\
2000 \\
6 \\
40 \\
2 \\
1 \\
2 \\
2 \mathrm{~d} \\
30\end{array}$ & $\begin{array}{r}1 \\
20 \\
1\end{array}$ & $\begin{array}{l}3804.534 \\
3806.144 \\
3806.424 \\
3807.381 \\
3808.633 \\
3809.798 \\
3810.355 \\
3810.568 \\
3811.253 \\
3812.390\end{array}$ & $\begin{array}{c}2 \\
15 \\
5 \\
10 \\
10 \mathrm{~d} \\
1 \\
2 \\
30 \\
80 \\
10\end{array}$ & 2 & $\begin{array}{l}3863.068 \\
3863.830 \\
3864.106 \\
3865.112 \\
3866.012 \\
3866.190 \\
3866.608 \\
3868.240 \\
3870.292 \\
3871.055\end{array}$ & $\begin{array}{r}200 \\
100 \\
400 \\
3 \\
30 \\
5 \\
5 \\
1000 \\
10 \\
12\end{array}$ & $\begin{array}{c}3 \\
1 \\
4 \\
\\
20 \mathrm{cw} \\
8\end{array}$ & $\begin{array}{l}3924.469 \\
3925.143 \\
3925.953 \\
3927.356 \\
3927.573 \\
3928.323 \\
3928.770 \\
3928.913 \\
3929.113 \\
3929.378\end{array}$ & $\begin{array}{r}2 \\
10 \\
20 \\
5 \\
200 \\
10 \\
30 \\
20 \\
10 \\
40\end{array}$ & 2 \\
\hline $\begin{array}{l}3768.774 \\
3769.352 \\
3769.680 \\
3769.783 \\
3770.320 \\
3770.498 \\
3771.031 \\
3771.467 \\
3772.088 \\
3772.388\end{array}$ & $\begin{array}{r}15 \\
3000 \\
4 \\
60 \\
40\end{array}$ & $\begin{array}{c}100 \\
1 \\
1 \mathrm{~h} \\
50 \\
2 \\
2\end{array}$ & $\begin{array}{l}3813.040 \\
3813.846 \\
3814.339 \\
3814.667 \\
3815.509 \\
3816.888 \\
3817.967 \\
3818.119 \\
3819.103 \\
3820.761\end{array}$ & $\begin{array}{c}15 \mathrm{cw} \\
10 \\
6 \\
200 \\
80 \\
300 \\
150 \\
100 \\
1 \\
50\end{array}$ & $\begin{array}{c}1 \mathrm{c} \\
2 \mathrm{~h} \\
3 \\
1 \\
4 \\
3 \\
2 \\
10 \mathrm{c} \\
2\end{array}$ & $\begin{array}{l}3871.223 \\
3874.609 \\
3875.658 \\
3876.366 \\
3877.139 \\
3878.295 \\
3879.160 \\
3880.715 \\
3882.497 \\
3885.638\end{array}$ & $\begin{array}{c}12 \\
12 \\
200 \\
80 \\
30 \\
4 \\
500 \mathrm{c} \\
600 \mathrm{c} \\
10 \\
3\end{array}$ & $\begin{array}{c}2 \\
1 \\
10 \mathrm{c} \\
20 \mathrm{c}\end{array}$ & $\begin{array}{l}3929.821 \\
3931.319 \\
3931.907 \\
3932.218 \\
3933.438 \\
3933.705 \\
3934.832 \\
3936.175 \\
3936.648 \\
3939.726\end{array}$ & $\begin{array}{r}5 \\
3 \\
8 \\
2 \\
150 \\
200 \\
30 \\
1 \\
70 \\
25\end{array}$ & 2 \\
\hline $\begin{array}{l}3772.782 \\
3773.413 \\
3773.930 \\
3774.783 \\
3774.970 \\
3775.355 \\
3775.560 \\
3776.288 \\
3776.999 \\
3777.274\end{array}$ & $\begin{array}{l}20 \\
50 \\
1 \\
15 \\
10 \\
50 \mathrm{c} \\
15 \\
1 \\
6 \mathrm{~h} \\
500\end{array}$ & $\begin{array}{l}1 \\
4\end{array}$ & $\begin{array}{l}3821.083 \\
3822.672 \\
3823.066 \\
3824.102 \\
3824.467 \\
3825.531 \\
3826.013 \\
3826.352 \\
3827.193 \\
3827.593\end{array}$ & $\begin{array}{r}4 \\
80 \\
1 \\
1 \\
300 \\
5 \\
20 \\
1 \\
2 \\
60\end{array}$ & 1 & $\begin{array}{l}3886.896 \\
3888.177 \\
3888.440 \\
3888.665 \\
3888.814 \\
3889.130 \\
3890.033 \\
3890.320 \\
3892.122 \\
3892.332\end{array}$ & $\begin{array}{c}2 \\
1 \mathrm{~h} \\
80 \\
7 \\
2 \\
150 \\
1 \\
20 \\
300 \mathrm{cw} \\
100\end{array}$ & $\begin{array}{c}1 \\
2 \\
100 \mathrm{cw} \\
1\end{array}$ & $\begin{array}{l}3940.054 \\
3940.478 \\
3942.866 \\
3943.868 \\
3946.571 \\
3947.087 \\
3948.151 \\
3948.513 \\
3948.844 \\
3949.224\end{array}$ & $\begin{array}{c}70 \\
30 \\
120 \\
4000 \mathrm{c} \\
2000 \\
1 \\
2 \\
4 \\
9\end{array}$ & $\begin{array}{l}2 \mathrm{~h} \\
1 \\
20 \mathrm{c} \\
10\end{array}$ \\
\hline $\begin{array}{l}3778.194 \\
3779.032 \\
3779.373 \\
3779.693 \\
3780.680 \\
3782.299 \\
3784.057 \\
3784.802 \\
3785.222 \\
3785.560\end{array}$ & $\begin{array}{c}10 \\
2000 \\
40 \\
3000 \mathrm{cw} \\
15 \\
500 \\
1 \\
20 \\
6\end{array}$ & $\begin{array}{c}2 \mathrm{hw} \\
40 \\
1 \\
100 \mathrm{cw} \\
6 \\
1\end{array}$ & $\begin{array}{l}3828.537 \\
3828.750 \\
3829.220 \\
3829.274 \\
3830.352 \\
3831.883 \\
3832.448 \\
3832.817 \\
3833.425 \\
3837.559\end{array}$ & $\begin{array}{r}500 \\
3 \\
150 \\
\\
200 \\
20 \\
200 \\
600 \\
\\
1500\end{array}$ & $\begin{array}{c}10 \mathrm{~h} \\
3 \\
\\
1 \\
5 \\
3 \mathrm{~h} \\
15\end{array}$ & $\begin{array}{l}3893.220 \\
3893.837 \\
3894.201 \\
3894.482 \\
3894.688 \\
3895.390 \\
3898.531 \\
3899.120 \\
3899.827 \\
3901.473\end{array}$ & $\begin{array}{r}200 \\
4 \\
15 \\
15 \\
2 \\
2 \\
2 \\
100 \\
600 \\
5\end{array}$ & $\begin{array}{l}1 \\
5\end{array}$ & $\begin{array}{l}3950.391 \\
3951.016 \\
3951.831 \\
3952.193 \\
3953.651 \\
3953.765 \\
3955.734 \\
3956.410 \\
3958.114 \\
3958.793\end{array}$ & $\begin{array}{r}80 \\
7 \\
180 \\
6 \\
100 \\
30 \\
200 \\
3 \\
10 \\
15\end{array}$ & $\begin{array}{l}1 \\
3\end{array}$ \\
\hline $\begin{array}{l}3786.064 \\
3786.494 \\
3786.566 \\
3787.537 \\
3787.772 \\
3788.154 \\
3788.370 \\
3789.232 \\
3789.955 \\
3791.282\end{array}$ & $\begin{array}{c}1 \\
2 \\
1 \\
2 \\
2 \mathrm{~h} \\
500\end{array}$ & $\begin{array}{l}3 \\
3 \mathrm{~h} \\
3 \mathrm{~h}\end{array}$ & $\begin{array}{l}3839.577 \\
3840.137 \\
3841.307 \\
3842.342 \\
3843.349 \\
3843.968 \\
3844.061 \\
3844.347 \\
3845.974 \\
3846.941\end{array}$ & $\begin{array}{r}1 \\
60 \\
800 \\
80 \\
2 \\
100 \\
20 \\
60 \\
800 \\
8\end{array}$ & $\begin{array}{l}2 \\
1 \\
8\end{array}$ & $\begin{array}{l}3902.832 \\
3903.740 \\
3903.934 \\
3904.195 \\
3905.322 \\
3905.517 \\
3905.774 \\
3906.400 \\
3907.681 \\
3907.958\end{array}$ & $\begin{array}{r}10 \\
60 \\
80 \\
3 \\
8 \\
30 \\
100 \\
20 \\
1 \\
30\end{array}$ & 1 & $\begin{array}{l}3959.090 \\
3959.520 \\
3959.943 \\
3960.770 \\
3961.520 \\
3963.361 \\
3963.714 \\
3964.266 \\
3964.467 \\
3965.619\end{array}$ & $\begin{array}{c}80 \mathrm{c} \\
150 \\
20 \mathrm{w} \\
2 \\
2 \\
30 \\
20 \\
3\end{array}$ & $\begin{array}{c}1 \mathrm{c} \\
2 \\
15 \mathrm{~W} \\
1\end{array}$ \\
\hline
\end{tabular}


TABLE I. Spectrum of technetium - Continued

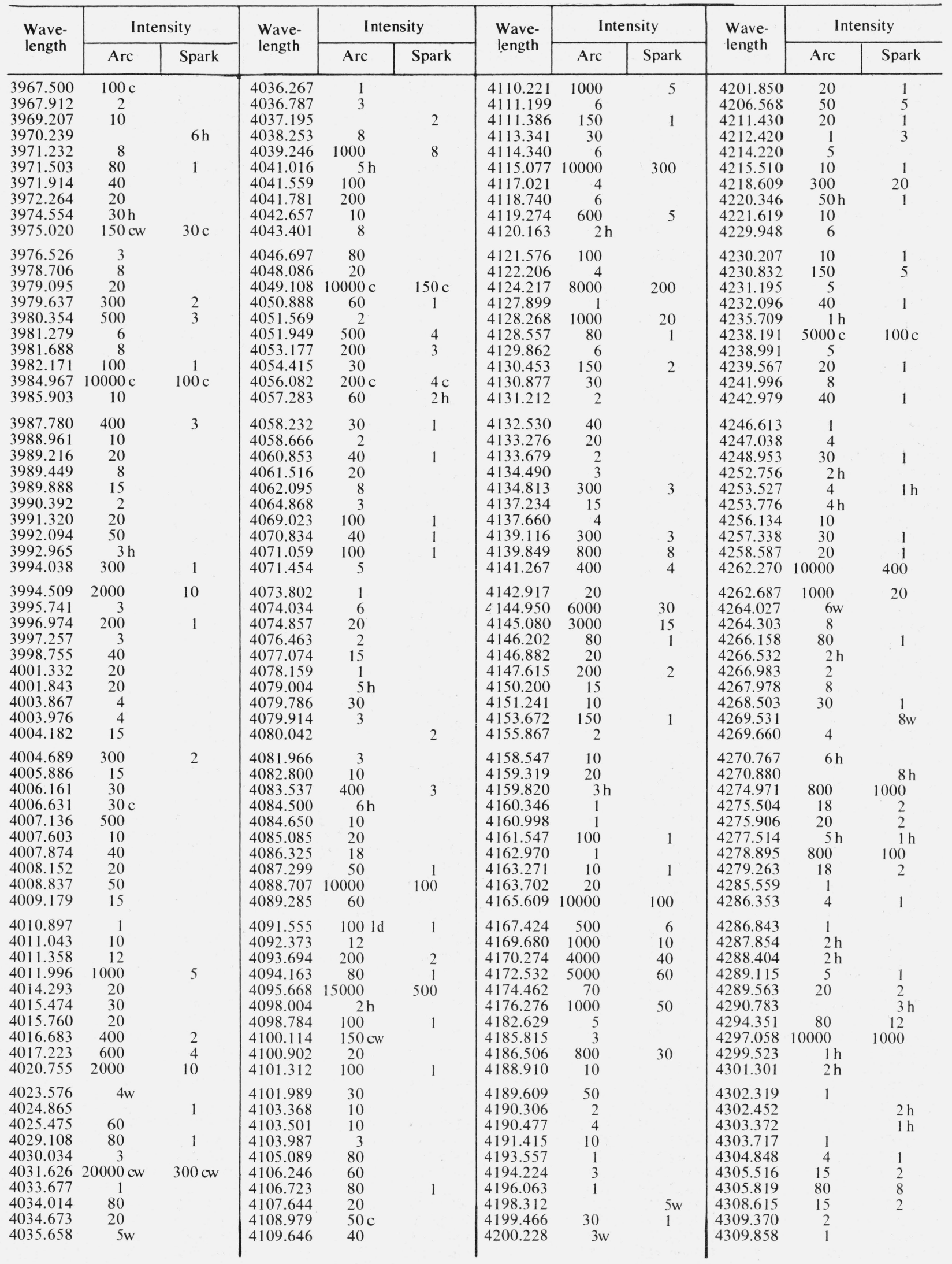


TABLE I. Spectrum of technetium - Continued

\begin{tabular}{|c|c|c|c|c|c|c|c|c|c|c|c|}
\hline $\begin{array}{l}\text { Wave- } \\
\text { length }\end{array}$ & \multicolumn{2}{|c|}{ Intensity } & $\begin{array}{l}\text { Wave- } \\
\text { length }\end{array}$ & \multicolumn{2}{|c|}{ Intensity } & $\begin{array}{l}\text { Wave- } \\
\text { length }\end{array}$ & \multicolumn{2}{|c|}{ Intensity } & $\begin{array}{l}\text { Wave- } \\
\text { length }\end{array}$ & \multicolumn{2}{|c|}{ Intensity } \\
\hline $\begin{array}{l}4310.222 \\
4311.376 \\
4312.504 \\
4314.021 \\
4314.679 \\
4318.061 \\
4318.983 \\
4319.887 \\
4320.505 \\
4321.068\end{array}$ & $\begin{array}{r}60 \\
3 \\
10 \\
9 \\
1 \\
6 \\
5 \\
2 \\
3 \\
1\end{array}$ & $\begin{array}{l}1 \\
1 \\
1 \\
1\end{array}$ & $\begin{array}{l}4395.571 \\
4397.254 \\
4398.003 \\
4398.689 \\
4399.337 \\
4401.518 \\
4402.054 \\
4402.819 \\
4403.905 \\
4404.176\end{array}$ & $\begin{array}{c}2 \\
1 \\
1 \\
4 \\
10 \\
1 \mathrm{~h} \\
\\
6 \\
2\end{array}$ & $\begin{array}{l}2 \mathrm{~h} \\
1\end{array}$ & $\begin{array}{l}4472.512 \\
4473.662 \\
4474.506 \\
4475.003 \\
4477.233 \\
4478.078 \\
4478.673 \\
4480.251 \\
4481.533 \\
4484.351\end{array}$ & $\begin{array}{r}2 \mathrm{~h} \\
15 \mathrm{c} \\
8 \\
7 \\
\\
2 \\
4 \\
100 \\
1000 \\
10\end{array}$ & $\begin{array}{r}2 \\
1 \\
1 \\
3 \\
1 \\
1 \\
10 \\
100 \\
1\end{array}$ & $\begin{array}{l}4547.088 \\
4548.614 \\
4549.156 \\
4549.439 \\
4550.270 \\
4551.728 \\
4552.204 \\
4552.456 \\
4552.854 \\
4554.042\end{array}$ & $\begin{array}{c}1 \\
1 \\
1 \mathrm{~h} \\
80 \\
4 \mathrm{~h} \\
2 \\
400 \\
150 \\
800 \\
1\end{array}$ & $\begin{array}{r}40 \\
15 \\
80 \\
3\end{array}$ \\
\hline $\begin{array}{l}4322.015 \\
4323.016 \\
4323.356 \\
4323.958 \\
4324.867 \\
4326.393 \\
4328.778 \\
4330.231 \\
4331.866 \\
4332.452\end{array}$ & $\begin{array}{r}2 \\
80 \\
12 \\
12 \\
3 \\
60 \\
2 \\
1 \\
150\end{array}$ & $\begin{array}{l}2 \mathrm{~h} \\
7 \\
2 \\
2 \\
1 \\
6\end{array}$ & $\begin{array}{l}4404.403 \\
4405.081 \\
4405.485 \\
4405.922 \\
4406.427 \\
4407.346 \\
4409.034 \\
4409.815 \\
4412.004 \\
4412.685\end{array}$ & $\begin{array}{r}1 \\
7 \\
2 \\
3 \\
1 \\
7 \\
3 \\
2 \\
100 \\
2\end{array}$ & $\begin{array}{l}1 \\
1 \\
7 \\
1\end{array}$ & $\begin{array}{l}4486.050 \\
4487.061 \\
4488.624 \\
4490.128 \\
4491.473 \\
4492.173 \\
4492.735 \\
4493.388 \\
4495.027 \\
4495.213\end{array}$ & $\begin{array}{c}6 \mathrm{c} \\
3000 \\
3 \\
1 \\
1 \mathrm{~h} \\
2 \\
10 \\
8 \mathrm{~h} \\
400 \\
20\end{array}$ & $\begin{array}{l}2 \mathrm{~h} \\
1 \\
7 \mathrm{~h} \\
50\end{array}$ & $\begin{array}{c}4557.046 \\
4558.711 \\
4560.205 \\
4560.421 \\
1562.522 \\
4562.913 \\
4563.741 \\
4564.539 \\
4565.436 \\
4566.421\end{array}$ & $\begin{array}{c}1000 \\
40 \\
15 \\
20 \mathrm{c} \\
8 \\
30 \\
25 \\
2000 \\
4 \\
7\end{array}$ & $\begin{array}{r}100 \\
4 \\
2 \\
2 \\
1 \\
3 \\
2 \\
300 \\
1 \\
1\end{array}$ \\
\hline $\begin{array}{l}4334.768 \\
4335.187 \\
4336.855 \\
4338.248 \\
4339.914 \\
4341.109 \\
4342.242 \\
4342.570 \\
4343.584 \\
4343.918\end{array}$ & $\begin{array}{c}3 \\
4 \\
400 \mathrm{cw} \\
2 \mathrm{~h} \\
3 \mathrm{~h} \\
2 \\
20 \\
4 \\
1 \\
4\end{array}$ & $\begin{array}{c}1 \\
1 \\
40 \mathrm{cw}\end{array}$ & $\begin{array}{l}4413.322 \\
4415.452 \\
4415.718 \\
4417.269 \\
4418.412 \\
4420.302 \\
4421.430 \\
4421.878 \\
4423.089 \\
4423.781\end{array}$ & $\begin{array}{c}1 \\
20 \mathrm{c} \\
5 \\
1 \\
7 \\
80 \\
2 \mathrm{~h} \\
3 \mathrm{~h} \\
2 \mathrm{~h} \\
3 \mathrm{hw}\end{array}$ & $\begin{array}{l}1 \\
1 \\
1 \\
6\end{array}$ & $\begin{array}{l}4497.337 \\
4497.713 \\
4498.529 \\
4499.295 \\
4500.170 \\
4501.743 \\
4503.399 \\
4504.058 \\
4505.081 \\
4505.473\end{array}$ & $\begin{array}{c}2 \mathrm{w} \\
5 \\
60 \\
7 \mathrm{~h} \\
1 \mathrm{~h} \\
60 \\
100 \\
1 \mathrm{~h} \\
1 \\
2\end{array}$ & $\begin{array}{l}8 \\
1 \mathrm{~h} \\
6 \\
6 \\
10\end{array}$ & $\begin{array}{l}4567.216 \\
4568.135 \\
4568.769 \\
4569.751 \\
4570.599 \\
4570.861 \\
4571.453 \\
4572.062 \\
4573.977 \\
4576.521\end{array}$ & $\begin{array}{l}1 \mathrm{~h} \\
3 \\
1 \\
1 \\
15 \\
60 \\
2 \\
5 \\
8 \\
2\end{array}$ & $\begin{array}{l}10 \\
1 \\
1\end{array}$ \\
\hline $\begin{array}{l}4344.579 \\
4345.442 \\
4350.013 \\
4350.415 \\
4352.487 \\
4354.823 \\
4355.398 \\
4356.093 \\
4358.491 \\
4359.257\end{array}$ & $\begin{array}{c}70 \\
10 \\
5 \mathrm{~h} \\
1 \\
2 \mathrm{~h} \\
2 \\
15 \mathrm{~W} \\
3 \\
400 \\
200\end{array}$ & $\begin{array}{r}2 \\
1 \\
40 \\
30\end{array}$ & $\begin{array}{l}4425.139 \\
4427.294 \\
4428.702 \\
4429.592 \\
4431.716 \\
4432.571 \\
4433.783 \\
4434.486 \\
4434.845 \\
4436.731\end{array}$ & $\begin{array}{c}1 \mathrm{~h} \\
10 \\
2 \\
1000 \\
15 \\
12 \\
1 \mathrm{~h} \\
1 \\
2\end{array}$ & $\begin{array}{r}1 \\
1 \\
200 \\
2 \\
2 \\
\\
20 \mathrm{c}\end{array}$ & $\begin{array}{l}4505.881 \\
4506.068 \\
4506.640 \\
4507.177 \\
4507.775 \\
4508.719 \\
4511.312 \\
4512.691 \\
4514.019 \\
4515.978\end{array}$ & $\begin{array}{c}3 \\
1 \\
2 \\
4 \\
3 \\
8 \\
3 \mathrm{~h} \\
8 \\
2 \mathrm{~h} \\
1000\end{array}$ & 1 & $\begin{array}{l}4577.129 \\
4578.447 \\
4579.084 \\
4579.559 \\
4580.291 \\
4580.613 \\
4582.377 \\
4582.726 \\
4583.246 \\
4583.686\end{array}$ & $\begin{array}{c}20 \\
1000 \\
60 \\
3 \\
3 \mathrm{w} \\
7 \\
25 \\
4 \\
1 \\
2\end{array}$ & $\begin{array}{r}3 \\
100 \\
5\end{array}$ \\
\hline $\begin{array}{l}4361.959 \\
4363.014 \\
4363.838 \\
4364.017 \\
4364.889 \\
4365.425 \\
4365.999 \\
4368.340 \\
4368.616 \\
4368.940\end{array}$ & $\begin{array}{r}20 \\
80 \\
\\
1 \\
1 \\
1 \\
4 \\
2 \\
1 \\
30\end{array}$ & $\begin{array}{l}2 \\
8 \\
1\end{array}$ & $\begin{array}{l}4437.056 \\
4438.999 \\
4441.418 \\
4445.316 \\
4446.502 \\
4447.015 \\
4450.790 \\
4451.433 \\
4452.078 \\
4452.273\end{array}$ & $\begin{array}{l}1 \mathrm{~h} \\
5 \\
2 \mathrm{~h} \\
6 \\
8 \\
12 \mathrm{c} \\
5 \mathrm{c} \\
1 \\
6 \\
60\end{array}$ & $\begin{array}{l}1 \\
2 \\
1\end{array}$ & $\begin{array}{l}4516.601 \\
4521.263 \\
4522.075 \\
4522.841 \\
4525.760 \\
4526.321 \\
4526.507 \\
4531.157 \\
4531.219 \\
4531.391\end{array}$ & $\begin{array}{r}20 \\
4 \\
10000 \\
2 \\
10 \\
8 \\
30 \\
5 \\
8\end{array}$ & $\begin{array}{c}2 \mathrm{~h} \\
2 \\
1 \\
1000 \\
\\
2 \\
1 \\
4 \\
1 \\
1\end{array}$ & $\begin{array}{l}4584.710 \\
4584.851 \\
4585.884 \\
4586.309 \\
4587.037 \\
4587.628 \\
4588.232 \\
4588.536 \\
4588.857 \\
4589.297\end{array}$ & $\begin{array}{c}3 \mathrm{~h} \\
50 \\
150 \\
4 \\
6 \\
4 \\
30 \\
2 \\
\\
2 \mathrm{~h}\end{array}$ & $\begin{array}{c}1 \mathrm{~h} \\
4 \\
15 \\
1 \\
1 \\
1 \\
3 \\
2 \\
3\end{array}$ \\
\hline $\begin{array}{l}4369.255 \\
4370.094 \\
4370.717 \\
4371.661 \\
4372.086 \\
4373.181 \\
4373.952 \\
4378.519 \\
4380.132 \\
4381.583\end{array}$ & $\begin{array}{r}25 \\
70 \\
60 \\
1 \\
10 \\
15 \\
70 \\
\\
2 \\
4\end{array}$ & $\begin{array}{l}3 \\
7 \\
7 \\
1 \\
2 \\
7 \\
2 \mathrm{~h} \\
1\end{array}$ & $\begin{array}{l}4452.581 \\
4454.142 \\
4454.811 \\
4456.621 \\
4458.057 \\
4459.395 \\
4463.038 \\
4463.535 \\
4463.852 \\
4464.277\end{array}$ & $\begin{array}{r}1 \\
3 \\
1 \\
20 \\
3 \\
10 \\
20 \\
1 \\
60 \\
15\end{array}$ & $\begin{array}{l}3 \\
2 \\
2 \\
6 \\
1\end{array}$ & $\begin{array}{l}4531.703 \\
4534.236 \\
4534.299 \\
4534.760 \\
4536.110 \\
4536.365 \\
4536.680 \\
4537.052 \\
4537.347 \\
4537.844\end{array}$ & $\begin{array}{c}20 \\
1 \\
1 \\
3 \mathrm{~h} \\
1 \\
2 \\
2 \\
2 \\
100 \\
5\end{array}$ & $\begin{array}{r}10 \\
1\end{array}$ & $\begin{array}{l}4590.690 \\
4591.689 \\
4591.988 \\
4592.306 \\
4593.346 \\
4594.059 \\
4597.273 \\
4597.393 \\
4598.217 \\
4600.164\end{array}$ & $\begin{array}{c}1 \mathrm{~h} \\
3 \mathrm{w} \\
1 \\
1 \\
1000 \\
100 \\
2 \mathrm{~h} \\
10 \\
1\end{array}$ & $\begin{array}{c}100 \\
10 \\
2 \mathrm{~h} \\
1\end{array}$ \\
\hline $\begin{array}{l}4383.075 \\
4384.317 \\
4384.574 \\
4385.626 \\
4385.881 \\
4387.501 \\
4388.605 \\
4391.923 \\
4392.265 \\
4393.787\end{array}$ & $\begin{array}{r}60 \\
1 \\
1 \\
1 \\
1 \\
15 \\
3 \\
3 \\
1 \\
7\end{array}$ & $\begin{array}{l}1 \\
1 \\
1\end{array}$ & $\begin{array}{l}4464.864 \\
4465.090 \\
4465.613 \\
4466.575 \\
4467.384 \\
4468.309 \\
4469.173 \\
4470.061 \\
4470.369 \\
4471.986\end{array}$ & $\begin{array}{c}6 \\
20 \\
80 \\
3 \\
3 \\
1 \mathrm{~h} \\
10 \\
4 \\
1 \\
3\end{array}$ & $\begin{array}{l}2 \\
8 \\
1 \\
1 \\
\\
1 \\
1\end{array}$ & $\begin{array}{l}4538.556 \\
4539.530 \\
4540.200 \\
4541.247 \\
4542.090 \\
4543.900 \\
4544.609 \\
4545.546 \\
4546.672 \\
4546.896\end{array}$ & $\begin{array}{r}3 \\
2000 \\
3 \\
5 \\
400 \\
5 \\
3 \\
50 \\
2 \\
2\end{array}$ & $\begin{array}{r}40 \\
1 \\
5\end{array}$ & $\begin{array}{l}4601.231 \\
4601.586 \\
4602.408 \\
4602.721 \\
4603.162 \\
4607.170 \\
4608.005 \\
4608.314 \\
4608.833 \\
4609.159\end{array}$ & $\begin{array}{r}3 \\
2 \\
30 \\
100 \\
15 \\
3 \\
80 \\
20 \\
8 \\
300 \mathrm{c}\end{array}$ & $\begin{array}{r}3 \\
10 \\
2 \\
\\
8 \\
2 \\
1 \\
30 \mathrm{c}\end{array}$ \\
\hline
\end{tabular}


TABLE I. Spectrum of technetium - Continued

\begin{tabular}{|c|c|c|c|c|c|c|c|c|c|c|c|}
\hline $\begin{array}{l}4610.531 \\
4611.171 \\
4611.386 \\
4611.811 \\
4613.089 \\
4616.857 \\
4617.447 \\
4618.192 \\
4619.725 \\
4620.048 \\
4621.129 \\
4621.458 \\
4622.691 \\
4622.950 \\
4624.122 \\
4624.961 \\
4625.628 \\
4625.753 \\
4626.557 \\
4626.803\end{array}$ & $\begin{array}{r}20 \\
40 \\
2 \\
3 \\
1 \\
1000 \\
20 \\
\\
1 \\
3 \\
30 \\
40 \\
200 \mathrm{c} \\
30 \mathrm{c} \\
3 \\
300 \\
5 \\
5 \\
20 \\
2\end{array}$ & $\begin{array}{c}20 \mathrm{c} \\
1 \mathrm{c} \\
1 \\
30 \\
1 \\
1\end{array}$ & $\begin{array}{l}4686.406 \\
4687.627 \\
4687.978 \\
4688.365 \\
4689.359 \\
4689.810 \\
4692.384 \\
4694.282 \\
4695.788 \\
4697.047 \\
4697.513 \\
4700.186 \\
4701.169 \\
4701.814 \\
4702.586 \\
4702.880 \\
4704.802 \\
4705.542 \\
4706.918 \\
4707.575\end{array}$ & $\begin{array}{c}150 \\
20 \\
100 \\
3 \mathrm{~h} \\
400 \\
150 \\
60 \\
300 \\
15 \\
3 \\
25 \mathrm{~h} \\
1 \\
2 \\
5 \\
1 \\
50 \\
15 \\
5 \\
1000 \\
20\end{array}$ & $\begin{array}{r}15 \\
1 \\
15 \\
40 \\
5 \\
2 \\
30 \\
3 \\
2 \mathrm{~h}\end{array}$ & \begin{tabular}{|l}
4762.362 \\
4763.931 \\
4764.796 \\
4764.987 \\
4765.381 \\
4766.305 \\
4770.363 \\
4771.543 \\
4773.237 \\
4773.890 \\
4776.355 \\
4777.557 \\
4778.820 \\
4779.501 \\
4779.898 \\
4780.607 \\
4781.012 \\
4783.925 \\
4784.137 \\
4784.720
\end{tabular} & $\begin{array}{c}200 \\
1 \\
5 \\
3 \\
8 \\
7 \\
1 \\
4000 \\
5 \\
200 \\
120 \\
1 \\
5 \\
8 \mathrm{c} \\
50 \\
80 \\
1 \mathrm{~h} \\
200 \\
20 \\
100\end{array}$ & $\begin{array}{r}1 \\
1 \\
400 \\
1 \\
20 \\
12 \\
1 \\
1 \mathrm{c} \\
5 \\
3 \\
\\
20 \\
2 \\
10\end{array}$ & $\begin{array}{l}4848.716 \\
4849.608 \\
4850.368 \\
4850.740 \\
4853.588 \\
4855.343 \\
4857.211 \\
4858.446 \\
4859.216 \\
4860.424 \\
4861.232 \\
4862.193 \\
4862.931 \\
4863.323 \\
4864.125 \\
4866.733 \\
4869.240 \\
4870.772 \\
4873.799 \\
4874.325\end{array}$ & $\begin{array}{c}3 \\
40 \\
4 \\
1 \mathrm{~h} \\
20000 \\
1 \\
100 \\
2 \\
40 \mathrm{c} \\
1 \\
20 \\
100 \mathrm{c} \\
40 \\
20 \\
6 \\
10000 \\
3 \mathrm{c} \\
200 \\
10 \mathrm{c} \\
1\end{array}$ & $\begin{array}{c}2000 \\
10 \\
2 \mathrm{c} \\
2 \\
8 \mathrm{c} \\
4 \\
2 \\
1 \\
1000\end{array}$ \\
\hline $\begin{array}{l}4628.063 \\
4629.253 \\
4630.572 \\
4630.978 \\
4631.378 \\
4631.815 \\
4632.740 \\
4633.152 \\
4635.433 \\
4635.752\end{array}$ & $\begin{array}{r}60 \\
1 \\
1000 \\
4 \\
80 \\
10 \\
30 \\
200 \\
1 \\
150\end{array}$ & $\begin{array}{r}3 \\
1 \\
3 \\
20 \\
\\
15\end{array}$ & $\begin{array}{l}4709.786 \\
4710.758 \\
4711.448 \\
4712.120 \\
4713.610 \\
4714.222 \\
4714.605 \\
4716.787 \\
4717.766 \\
4719.018\end{array}$ & $\begin{array}{c}1 \\
2 \\
2 \\
8 \mathrm{c} \\
3 \\
200 \\
40 \\
80 \\
2000 \\
500 \mathrm{c}\end{array}$ & $\begin{array}{r}20 \\
4 \\
8 \\
200 \\
50 \mathrm{c}\end{array}$ & $\begin{array}{l}4785.597 \\
4787.589 \\
4787.758 \\
4789.492 \\
4790.482 \\
4791.623 \\
4792.120 \\
4796.176 \\
4796.480 \\
4797.895\end{array}$ & $\begin{array}{c}500 \\
40 \\
100 \\
150 \\
200 \mathrm{c} \\
250 \\
5 \\
3 \\
10 \\
6 \mathrm{~h}\end{array}$ & $\begin{array}{c}50 \\
2 \\
8 \\
15 \\
20 \mathrm{c} \\
20 \\
1 \\
1\end{array}$ & $\begin{array}{l}4874.768 \\
4875.142 \\
4876.444 \\
4876.768 \\
4877.016 \\
4877.344 \\
4878.094 \\
4882.586 \\
4885.044 \\
44885.889\end{array}$ & $\begin{array}{r}30 \\
1 \\
1 \\
12 \mathrm{c} \\
30 \\
15 \\
12 \\
8 \\
30 \\
12\end{array}$ & $\begin{array}{l}1 \\
3 \\
2 \\
1 \\
1 \\
3 \\
1\end{array}$ \\
\hline $\begin{array}{l}4637.500 \\
4640.015 \\
4641.296 \\
4641.669 \\
4643.280 \\
4645.490 \\
4646.620 \\
4647.573 \\
4648.332 \\
4650.556\end{array}$ & $\begin{array}{c}3000 \\
2 \\
6 \\
1 \\
500 \\
150 \mathrm{c} \\
80 \\
120 \\
2000 \\
8\end{array}$ & $\begin{array}{c}300 \\
1 \\
20 \\
5 \mathrm{c} \\
8 \\
10 \\
200 \\
1\end{array}$ & $\begin{array}{l}4719.281 \\
4720.726 \\
4723.828 \\
4724.842 \\
4725.202 \\
4726.798 \\
4728.082 \\
4729.302 \\
4731.023 \\
4731.918\end{array}$ & $\begin{array}{c}4000 \mathrm{c} \\
6 \\
3 \\
1 \\
1 \\
10 \\
50 \\
15 \\
2 \mathrm{cw} \\
10\end{array}$ & $\begin{array}{c}400 \mathrm{c} \\
1 \\
\\
1 \\
5 \\
2 \\
1\end{array}$ & $\begin{array}{l}4799.079 \\
4799.978 \\
4800.700 \\
4803.291 \\
4803.623 \\
4804.777 \\
4805.688 \\
4806.992 \\
4807.349 \\
4807.988\end{array}$ & $\begin{array}{c}4 \\
300 \\
80 \mathrm{c} \\
30 \\
2 \\
1 \mathrm{~h} \\
100 \\
8 \\
8 \\
1 \mathrm{~h}\end{array}$ & $\begin{array}{l}30 \\
5 \mathrm{c}\end{array}$ & $\begin{array}{l}4886.190 \\
4888.695 \\
4889.444 \\
4890.884 \\
4891.918 \\
4892.490 \\
4893.153 \\
4893.651 \\
4894.780 \\
4895.977\end{array}$ & $\begin{array}{c}4 \\
100 \\
50 \\
150 \mathrm{c} \\
8000 \\
150 \\
1 \mathrm{~h} \\
10 \mathrm{c} \\
5 \mathrm{cW} \\
2\end{array}$ & $\begin{array}{c}10 \\
5 \\
10 \mathrm{c} \\
800 \\
15 \\
1 \mathrm{c} \\
20 \mathrm{cw}\end{array}$ \\
\hline $\begin{array}{l}4652.005 \\
4653.609 \\
4654.658 \\
4655.363 \\
4656.440 \\
4657.006 \\
4658.934 \\
4659.798 \\
4660.212 \\
4660.590\end{array}$ & $\begin{array}{c}2 \\
100 \\
120 \\
1 \\
12 \\
60 \mathrm{sd} \\
2 \\
30 \\
2000 \mathrm{c} \\
30 \mathrm{c}\end{array}$ & $\begin{array}{r}1 \\
10 \\
1 \\
6 \\
3 \\
200 \\
3\end{array}$ & $\begin{array}{l}4732.044 \\
4732.469 \\
4733.869 \\
4735.058 \\
4736.515 \\
4737.434 \\
4738.652 \\
4739.551 \\
4740.608 \\
4741.284\end{array}$ & $\begin{array}{c}10 \\
25 \\
20 \mathrm{c} \\
8 \\
200 \mathrm{~cd} \\
1 \\
2 \\
100 \mathrm{c} \\
10000 \\
50\end{array}$ & $\begin{array}{c}1 \\
1 \\
2 \mathrm{c} \\
1 \\
20 \mathrm{~cd}\end{array}$ & $\begin{array}{l}4809.094 \\
4809.416 \\
4810.633 \\
4811.842 \\
4812.630 \\
4813.324 \\
4814.073 \\
4816.062 \\
4816.794 \\
4819.024\end{array}$ & $\begin{array}{c}1 \\
100 \\
2 \\
60 \\
3 \\
50 \\
60 \\
30 \mathrm{c} \\
500 \\
1 \mathrm{~h}\end{array}$ & $\begin{array}{r}10 \\
6 \\
\\
5 \\
6 \\
1 \\
80\end{array}$ & $\begin{array}{l}4896.832 \\
4897.251 \\
4899.704 \\
4900.046 \\
4900.955 \\
4901.708 \\
4902.085 \\
4903.747 \\
4906.410 \\
4906.791\end{array}$ & $\begin{array}{r}1 \\
8 \\
1 \\
30 \\
10 \\
20 \\
1 \\
2 \\
50 \\
10\end{array}$ & $\begin{array}{l}1 \\
3 \\
1 \\
2\end{array}$ \\
\hline $\begin{array}{l}4663.237 \\
4664.337 \\
4666.311 \\
4667.326 \\
4668.354 \\
4668.879 \\
4669.305 \\
4671.749 \\
4672.170 \\
4672.545\end{array}$ & $\begin{array}{r}2 \mathrm{~h} \\
100 \\
3 \\
1 \\
40 \\
100 \\
2000 \\
10 \\
400 \\
3\end{array}$ & $\begin{array}{r}10 \\
200 \\
1 \\
40\end{array}$ & $\begin{array}{l}4741.532 \\
4742.940 \\
4744.250 \\
4746.009 \\
4747.833 \\
4749.614 \\
4750.552 \\
4750.759 \\
4751.548 \\
4752.717\end{array}$ & $\begin{array}{c}6 \\
8 \\
1 \mathrm{~h} \\
100 \mathrm{csd} \\
1 \\
500 \\
3 \\
8 \\
15 \\
1000\end{array}$ & $\begin{array}{c}1 \\
1 \\
10 \mathrm{c} \mathrm{sd} \\
20 \\
1 \\
2 \\
60\end{array}$ & $\begin{array}{l}4820.739 \\
4825.236 \\
4825.748 \\
4828.203 \\
4828.698 \\
4831.347 \\
4834.367 \\
4835.394 \\
4838.034 \\
4839.017\end{array}$ & $\begin{array}{r}10000 \\
1 \\
20 \\
1 \\
2 \\
300 \\
1000 \\
1000 \\
1 \\
1\end{array}$ & $\begin{array}{r}1000 \\
2\end{array}$ & $\begin{array}{l}4908.509 \\
4909.053 \\
4909.566 \\
4912.422 \\
4913.020 \\
4914.166 \\
4914.699 \\
4915.372 \\
4915.810 \\
4918.917\end{array}$ & $\begin{array}{c}1000 \\
5 \\
2000 \\
5 \mathrm{c} \\
500 \\
10 \\
150 \mathrm{c} \\
1 \\
30 \\
3\end{array}$ & $\begin{array}{r}100 \\
1 \\
200 \\
\\
50 \\
1 \\
15 \mathrm{c} \\
3\end{array}$ \\
\hline $\begin{array}{l}4672.824 \\
4673.077 \\
4674.245 \\
4678.073 \\
4678.903 \\
4682.129 \\
4682.732 \\
4683.599 \\
4684.716 \\
4685.423\end{array}$ & $\begin{array}{c}40 \\
45 \\
30 \\
3 \\
200 \\
1 \mathrm{~h} \\
20 \mathrm{c} \\
150 \\
2 \\
1\end{array}$ & $\begin{array}{c}4 \\
4 \\
1 \\
\\
20 \\
1 \mathrm{~h} \\
1 \\
15\end{array}$ & $\begin{array}{l}4755.362 \\
4756.111 \\
4756.498 \\
4757.502 \\
4757.840 \\
4758.094 \\
4759.280 \\
4759.806 \\
4760.160 \\
4761.968\end{array}$ & $\begin{array}{c}1 \mathrm{~h} \\
100 \\
1 \\
40 \mathrm{c} \\
2 \\
80 \\
40 \\
50 \\
2 \\
40\end{array}$ & $\begin{array}{l}10 \\
2 \\
\\
6 \\
3 \\
3 \\
3\end{array}$ & $\begin{array}{l}4840.974 \\
4841.364 \\
4842.022 \\
4842.488 \\
4843.559 \\
4843.850 \\
4844.326 \\
4845.642 \\
4845.980 \\
4846.444\end{array}$ & $\begin{array}{c}20 \\
100 \\
10 \mathrm{~h} \\
1 \\
6 \\
1 \mathrm{~h} \\
2 \\
2 \\
1 \\
1\end{array}$ & $\begin{array}{c}2 \\
10 \\
1 \mathrm{~h} \\
1 \\
1\end{array}$ & $\begin{array}{l}4919.229 \\
4920.668 \\
4921.646 \\
4922.482 \\
4923.601 \\
4925.412 \\
4927.031 \\
4928.676 \\
4929.749 \\
4930.360\end{array}$ & $\begin{array}{c}5 \\
200 \mathrm{cl} \\
20 \\
2 \mathrm{w} \\
300 \\
2 \\
5 \\
3 \\
25 \\
20\end{array}$ & $\begin{array}{c}20 \mathrm{c} \\
2 \\
3 \\
1 \\
2 \\
2\end{array}$ \\
\hline
\end{tabular}


TABLE I. Spectrum of technetium - Continued

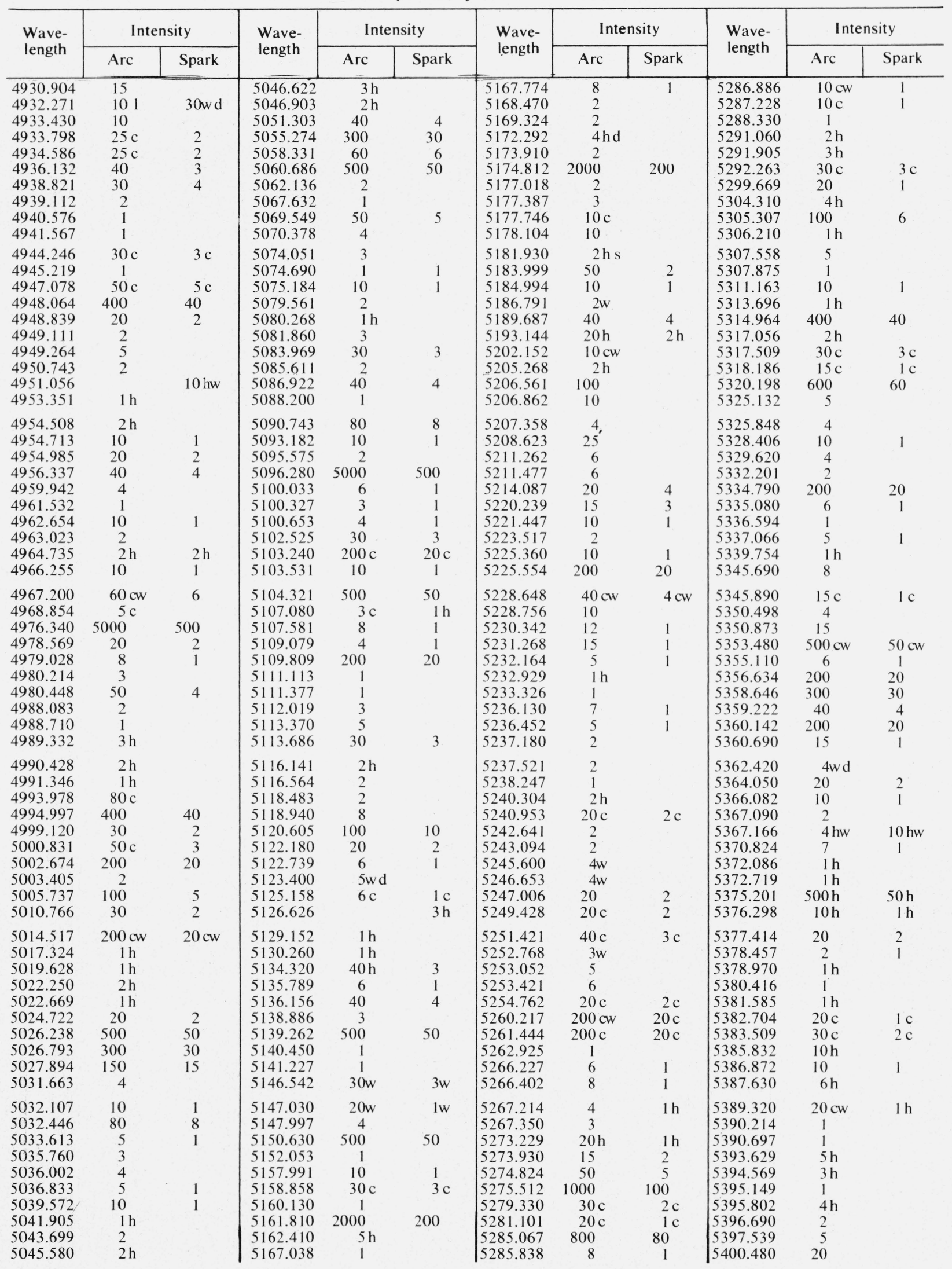


TABLE I. Spectrum of technetium - Continued

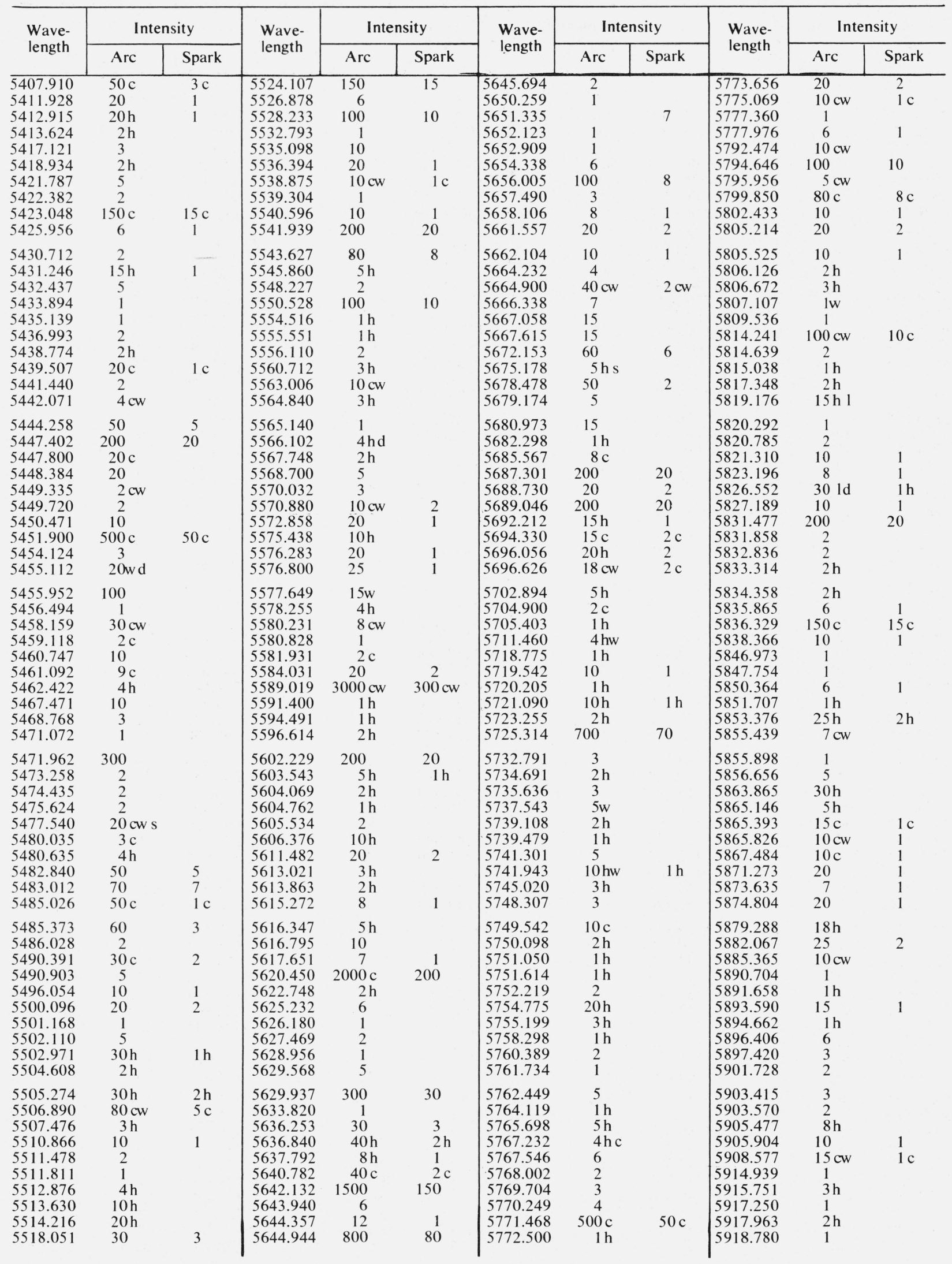


TABLE I. Spectrum of technetium - Continued

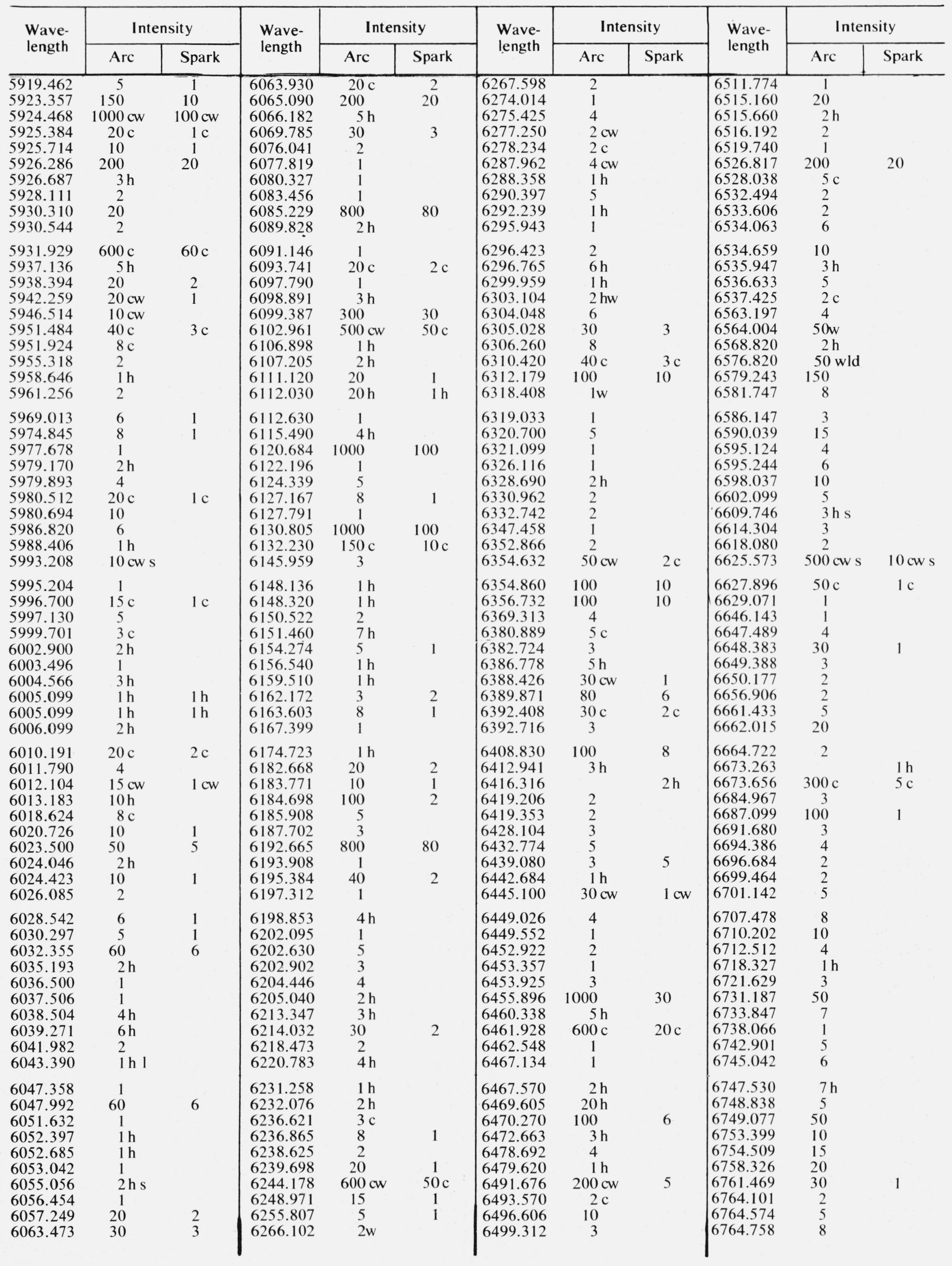


TABLE I. Spectrum of technetium - Continued

\begin{tabular}{|c|c|c|c|c|c|c|c|c|c|c|c|}
\hline $\begin{array}{l}\text { Wave- } \\
\text { length }\end{array}$ & Arc & Spark & $\begin{array}{l}\text { Wave- } \\
\text { length }\end{array}$ & Arc & Spark & $\begin{array}{l}\text { Wave- } \\
\text { length }\end{array}$ & Arc & Spark & $\begin{array}{l}\text { Wave- } \\
\text { length }\end{array}$ & Arc & Spark \\
\hline $\begin{array}{l}6767.183 \\
6770.387 \\
6772.562 \\
6773.899 \\
6776.580 \\
6781.305 \\
6786.003 \\
6786.452 \\
6786.965 \\
6787.951\end{array}$ & $\begin{array}{c}2 \\
1 \mathrm{~h} \\
1 \mathrm{~h} \\
4 \\
8 \\
1 \mathrm{~h} \\
80 \\
1 \\
10 \\
2\end{array}$ & 2 & $\begin{array}{l}6998.653 \\
7002.37 \\
7004.78 \\
7006.29 \\
7011.44 \\
7012.56 \\
7014.10 \\
7016.57 \\
7020.02 \\
7022.91\end{array}$ & $\begin{array}{c}15 \\
150 \\
6 \\
20 \mathrm{c} \\
2 \\
3 \mathrm{~h} \\
10 \mathrm{c} \\
100 \\
20 \\
5\end{array}$ & & \begin{tabular}{|l}
7350.29 \\
7360.06 \\
7362.46 \\
7366.33 \\
7395.06 \\
7396.80 \\
7402.61 \\
7405.36 \\
7421.12 \\
7424.01
\end{tabular} & $\begin{array}{c}1 \\
10 \\
5 \mathrm{w} \\
40 \mathrm{cw} \\
50 \\
100 \\
100 \mathrm{cw} 1 \\
200 \\
8 \\
20\end{array}$ & & \begin{tabular}{|l|}
7782.99 \\
7793.04 \\
7795.70 \\
7798.28 \\
7807.20 \\
7811.10 \\
7812.65 \\
7816.74 \\
7817.72 \\
7826.31
\end{tabular} & $\begin{array}{c}15 \\
800 \mathrm{cw} \\
2 \\
60 \\
10 \\
2 \\
4 \\
60 \\
800 \\
10\end{array}$ & \\
\hline $\begin{array}{l}6788.665 \\
6795.564 \\
6798.629 \\
6800.793 \\
6805.049 \\
6805.942 \\
6813.340 \\
6813.590 \\
6815.382 \\
6818.126\end{array}$ & $\begin{array}{r}3 \\
2 \\
70 \\
2 \\
5 \\
15 \\
5 \\
5 \\
1 \\
10\end{array}$ & 2 & $\begin{array}{l}7023.63 \\
7029.72 \\
7032.89 \\
7045.97 \\
7048.47 \\
7054.80 \\
7061.63 \\
7065.59 \\
7071.44 \\
7076.77\end{array}$ & $\begin{array}{r}6 \\
8 \\
10 \\
3 \\
30 \\
10 \\
7 \\
10 \\
30 \\
15\end{array}$ & & $\begin{array}{l}7427.15 \\
7429.12 \\
7431.65 \\
7434.12 \\
7452.49 \\
7461.59 \\
7470.14 \\
7471.77 \\
7494.28 \\
7512.28\end{array}$ & $\begin{array}{r}60 \\
1 \\
2 \\
150 \\
600 \\
60 \\
50 \\
20 \\
6 \\
3\end{array}$ & & $\begin{array}{l}7826.91 \\
7848.36 \\
7854.40 \\
7856.38 \\
7858.25 \\
7861.44 \\
7863.22 \\
7871.25 \\
7874.76 \\
7876.19\end{array}$ & $\begin{array}{c}10 \\
2 \mathrm{c} \\
10 \\
100 \\
10 \\
200 \\
10 \\
400 \mathrm{ld} \\
60 \mathrm{cw} \\
2\end{array}$ & \\
\hline $\begin{array}{l}6823.962 \\
6825.480 \\
6826.146 \\
6826.686 \\
6830.007 \\
6833.736 \\
6837.906 \\
6854.469 \\
6855.365 \\
6856.902\end{array}$ & $\begin{array}{l}1 \\
3 \\
5 \\
5 \\
8 \\
3 \\
4 \mathrm{hw} \\
3 \mathrm{~h} \\
5 \\
60\end{array}$ & 1 & $\begin{array}{l}7077.56 \\
7083.59 \\
7084.87 \\
7086.18 \\
7093.12 \\
7097.24 \\
7109.81 \\
7115.78 \\
7117.17 \\
7117.39\end{array}$ & $\begin{array}{c}2 \\
15 \\
1 \\
500 \mathrm{cw} \mathrm{d} \\
60 \\
3 \\
8 \\
8 \\
4 \\
5\end{array}$ & & $\begin{array}{l}7516.40 \\
7534.95 \\
7540.26 \\
7543.39 \\
7545.51 \\
7547.26 \\
7548.44 \\
7550.99 \\
7554.48 \\
7555.35\end{array}$ & $\begin{array}{c}3 w \\
80 \\
800 \\
80 \\
30 \\
5 \\
50 \\
10 w \\
5 \\
2\end{array}$ & & $\begin{array}{l}7879.37 \\
7881.63 \\
7885.98 \\
7894.75 \\
7898.56 \\
7905.72 \\
7911.29 \\
7912.19 \\
7920.37 \\
7949.71\end{array}$ & $\begin{array}{c}8 \mathrm{c} \\
5 \\
40 \mathrm{cw} \\
2 \\
3 \\
20 \mathrm{c} \\
4 \mathrm{c} \\
4 \mathrm{c} \\
10 \mathrm{~h} \\
15 \mathrm{c}\end{array}$ & \\
\hline $\begin{array}{l}6860.726 \\
6862.371 \\
6866.473 \\
6866.677 \\
6867.526 \\
6871.646 \\
6872.303 \\
6873.070 \\
6874.626 \\
6876.690\end{array}$ & $\begin{array}{r}30 \\
15 \\
15 \\
25 \\
2 \\
2 \\
1 \\
2 \\
10 \\
50\end{array}$ & 1 & $\begin{array}{l}7123.97 \\
7124.78 \\
7134.82 \\
7140.18 \\
7141.28 \\
7144.67 \\
7156.87 \\
7157.62 \\
7169.73 \\
7173.04\end{array}$ & $\begin{array}{c}4 \\
30 \\
1 \\
3 \\
200 \\
15 \\
20 \\
200 \mathrm{c} \\
2 \mathrm{w} \\
3\end{array}$ & & $\begin{array}{l}7574.02 \\
7577.61 \\
7579.26 \\
7583.93 \\
7584.33 \\
7586.44 \\
7587.56 \\
7588.61 \\
7593.15 \\
7600.96\end{array}$ & $\begin{array}{r}200 \\
2 \\
500 \\
5 \\
3 \\
5 \\
5 \\
7 \\
7 \\
3\end{array}$ & & $\begin{array}{l}7958.09 \\
7960.65 \\
7963.92 \\
7965.45 \\
7968.30 \\
7981.80 \\
7999.73 \\
8001.88 \\
8014.78 \\
8017.22\end{array}$ & $\begin{array}{c}5 \\
1 \\
20 \\
70 \\
50 \\
50 \mathrm{cw} \\
500 \\
10 \\
1 \\
8\end{array}$ & \\
\hline $\begin{array}{l}6933.236 \\
6939.892 \\
6941.465 \\
6944.988 \\
6945.597 \\
6949.320 \\
6951.282 \\
6952.625 \\
6958.593 \\
6960.046\end{array}$ & $\begin{array}{c}30 \\
4 \\
4 \\
2 \\
15 \\
7 \mathrm{~h} \\
3 \\
3 \\
4 \\
2\end{array}$ & & $\begin{array}{l}7243.35 \\
7248.12 \\
7251.68 \\
7253.09 \\
7256.08 \\
7264.84 \\
7283.16 \\
7296.24 \\
7304.60 \\
7305.00\end{array}$ & $\begin{array}{c}3 \mathrm{w} \\
4 \\
15 \\
5 \\
70 \\
3 \\
15 \\
5 \mathrm{wd} \\
6 \\
5\end{array}$ & & $\begin{array}{l}7655.82 \\
7657.04 \\
7658.37 \\
7661.98 \\
7667.64 \\
7672.72 \\
7676.95 \\
7679.20 \\
7681.79 \\
7684.45\end{array}$ & $\begin{array}{c}1 \\
2 \\
30 \mathrm{c} \\
1 \\
2 \\
2 \\
4 \\
2 \\
15 \\
100\end{array}$ & & $\begin{array}{l}8112.77 \\
8113.54 \\
8116.91 \\
8119.86 \\
8126.55 \\
8127.78 \\
8132.65 \\
8134.10 \\
8142.63 \\
8153.48\end{array}$ & $\begin{array}{c}1 \\
1 \\
5 \\
40 \\
200 \\
15 \\
5 \mathrm{~h} \\
3 \mathrm{~h} \\
30 \\
8\end{array}$ & \\
\hline $\begin{array}{l}6968.151 \\
6969.394 \\
6971.534 \\
6974.191 \\
6975.067 \\
6980.153 \\
6985.402 \\
6988.946 \\
6990.300 \\
6998.037\end{array}$ & $\begin{array}{r}30 \\
4 \\
1 \\
2 \\
7 \\
10 \\
30 \\
1 \\
40 \\
8\end{array}$ & & $\begin{array}{l}7316.44 \\
7320.30 \\
7322.38 \\
7327.32 \\
7329.14 \\
7331.89 \\
7338.20 \\
7340.13 \\
7341.85 \\
7345.81\end{array}$ & $\begin{array}{c}15 \mathrm{~d} \\
5 \mathrm{~W} \\
100 \\
4 \mathrm{~h} \\
80 \\
5 \\
50 \\
4 \\
2 \\
4\end{array}$ & & $\begin{array}{l}7697.37 \\
7698.19 \\
7701.92 \\
7719.59 \\
7722.19 \\
7733.63 \\
7737.36 \\
7746.60 \\
7777.22 \\
7779.59\end{array}$ & $\begin{array}{c}500 \\
80 \mathrm{cw} \\
1 \\
2 \mathrm{~h} \\
3 \\
5 \mathrm{c} \\
50 \mathrm{c} \\
10 \\
40 \\
6 \mathrm{c}\end{array}$ & & $\begin{array}{l}8160.53 \\
8160.94 \\
8170.55 \\
8175.13 \\
8176.18 \\
8200.05 \\
8205.27 \\
8206.49 \\
8208.41 \\
8211.31\end{array}$ & $\begin{array}{r}10 \mathrm{c} \\
15 \\
200 \\
1 \\
5 \\
3 \\
150 \\
100 \\
2 \\
150\end{array}$ & \\
\hline
\end{tabular}


TABLE 1. Spectrum of technetium-Continued

\begin{tabular}{|c|c|c|c|c|c|c|c|c|c|c|c|}
\hline \multirow{2}{*}{$\begin{array}{l}\text { Wave- } \\
\text { length }\end{array}$} & \multicolumn{2}{|c|}{ Intensity } & \multirow{2}{*}{$\begin{array}{l}\text { Wave- } \\
\text { length }\end{array}$} & \multicolumn{2}{|c|}{ Intensity } & \multirow{2}{*}{$\begin{array}{l}\text { Wave- } \\
\text { length }\end{array}$} & \multicolumn{2}{|c|}{ Intensity } & \multirow{2}{*}{$\begin{array}{l}\text { Wave- } \\
\text { length }\end{array}$} & \multicolumn{2}{|c|}{ Intensity } \\
\hline & Arc & Spark & & Arc & Spark & & Arc & Spark & & Arc & Spark \\
\hline $\begin{array}{l}8225.07 \\
8231.21 \\
8237.08 \\
8248.96 \\
8250.43 \\
8254.50 \\
8264.13 \\
8265.64 \\
8269.84 \\
8270.83\end{array}$ & $\begin{array}{c}50 \\
2 \\
500 \mathrm{cw} \\
40 \\
3 \\
30 \\
10 \\
3 \\
1 \mathrm{~h} \\
2 \mathrm{~h}\end{array}$ & & $\begin{array}{l}8309.16 \\
8315.50 \\
8319.07 \\
8322.49 \\
8323.35 \\
8330.37 \\
8346.57 \\
8383.67 \\
8399.36 \\
8404.00\end{array}$ & $\begin{array}{c}200 \\
60 \\
20 \mathrm{cw} \\
5 \\
1 \\
1 \\
40 \\
40 \\
1 \\
7\end{array}$ & & $\begin{array}{l}8497.12 \\
8507.15 \\
8514.70 \\
8519.73 \\
8522.53 \\
8531.06 \\
8537.65 \\
8543.61 \\
8595.93 \\
8604.09\end{array}$ & $\begin{array}{r}5 \mathrm{~h} \\
1 \\
30 \\
1 \\
20 \\
100 \\
30 \\
100 \\
10 \\
3\end{array}$ & & $\begin{array}{l}8735.34 \\
8737.93 \\
8751.77 \\
8756.86 \\
8757.41 \\
8766.68 \\
8787.24 \\
8790.90 \\
8808.81 \\
8812.42\end{array}$ & $\begin{array}{c}3 \\
100 \mathrm{cw} \\
20 \\
15 \\
4 \mathrm{w} \\
6 \\
3 \\
2 \\
2 \mathrm{~h} \\
5 \mathrm{c}\end{array}$ & \\
\hline $\begin{array}{l}8271.93 \\
8276.20 \\
8278.00 \\
8283.27 \\
8285.09 \\
8285.68 \\
8306.22 \\
8308.15\end{array}$ & $\begin{array}{l}2 \mathrm{hd} \\
1 \mathrm{~h} \\
1 \\
1 \\
1 \\
2 \\
2 \\
200\end{array}$ & & $\begin{array}{l}8415.55 \\
8422.43 \\
8445.82 \\
8462.80 \\
8477.91 \\
8481.34 \\
8484.00 \\
8492.13\end{array}$ & $\begin{array}{l}50 \mathrm{cw} \\
5 \\
20 \\
10 \mathrm{c} \\
2 \\
50 \mathrm{c} \\
40 \\
1\end{array}$ & & $\begin{array}{l}8639.39 \\
8645.03 \\
8652.76 \\
8664.12 \\
8673.62 \\
8707.21 \\
8719.95 \\
8722.68\end{array}$ & $\begin{array}{c}4 \\
3 \\
50 \\
6 \\
8 \\
100 \mathrm{cw} \\
30 \\
25\end{array}$ & & $\begin{array}{l}8820.91 \\
8829.82 \\
8897.64 \\
8901.47 \\
8909.93 \\
8917.73\end{array}$ & $\begin{array}{c}10 \mathrm{c} \\
200 \mathrm{cw} \\
2 \mathrm{c} \\
30 \mathrm{c} \\
2 \\
8\end{array}$ & \\
\hline
\end{tabular}

\section{References}

[1] C. Perrier and E. Segré, Nature 159, 24 (1947).

[2] W. F. Meggers and B. F. Scribner, J. Res. NBS 45, 476 (1950) [8] ibid. p. 19. RP2161.

[3] W. F. Meggers, J. Res. NBS 47, 7 (1951) RP2221.

[4] P. W. Merrill, Astrophys. J. 1 16, 21 (1952).

[5] W. R. Bozman, J. Opt. Soc. Am. 44, 824(A) (1954).
[6] W. R. Bozman, J. Opt. Soc. Am. 46, 383(A) (1956).

[7] C. E. Moore, Atomic Energy Levels, Vol III, NBS Circ. 467 (Washington, 1958) p. 17. 